\title{
TIME PROBLEM IN QUANTUM MECHANICS AND ITS ANALYSIS BY THE CONCEPT OF WEAK MEASUREMENTS
}

\author{
J. Ruseckas and B. Kaulakys \\ Vilnius University Research Institute of Theoretical Physics and Astronomy, A. Goštauto 12, LT-01108 Vilnius, Lithuania
}

Received 31 May 2004

Dedicated to the 100th anniversary of Professor A. Jucys

\begin{abstract}
The model of weak measurements is applied to various problems, related to the time problem in quantum mechanics. The review and generalization of the theoretical analysis of the time problem in quantum mechanics based on the concept of weak measurements are presented. A question of the time interval the system spends in the specified state, when the final state of the system is given, is raised. Using the concept of weak measurements the expression for such time is obtained. The results are applied to the tunnelling problem. A procedure for the calculation of the asymptotic tunnelling and reflection times is proposed. Examples for $\delta$-form and rectangular barrier illustrate the obtained results. Using the concept of weak measurements the arrival time probability distribution is defined by analogy with the classical mechanics. The proposed procedure is suitable to the free particles and to particles subjected to an external potential, as well. It is shown that such an approach imposes an inherent limitation to the accuracy of the arrival time definition.
\end{abstract}

Keywords: quantum measurement, tunnelling time, arrival time, quantum jumps

PACS: $03.65 . \mathrm{Xp}, 03.65 . \mathrm{Ta}, 42.50 . \mathrm{Lc}$

\section{Introduction}

Time plays a special role in quantum mechanics. Unlike other observables, time remains a classical variable. It cannot be simply quantized because, as it is well known, the self-adjoint operator of time does not exist for the bounded Hamiltonians. The problems related to time also arise from the fact that in quantum mechanics many quantities cannot have definite values simultaneously. The absence of the time operator makes this problem even more complicated. However, in practice time is often important for an experimenter. If quantum mechanics can correctly describe the outcomes of the experiments, it must also give the method for the calculation of the time the particle spends in some region.

The most-known problem of time in quantum mechanics is the so-called "tunnelling time problem". Tunnelling phenomena are inherent in numerous quantum systems, ranging from an atom and condensed matter to quantum fields. There have been many attempts to define a physical time for tunnelling processes, since this question has been raised by MacColl [1] in 1932. This question is still a subject of much controversy, since numerous theories contradict each other in their predictions for the "tunnelling time". Some of these theories predict that the tunnelling process is faster than light, whereas the others state that it should be subluminal. This subject has been covered in a number of reviews (by Hauge and Støvneng in 1989 [2], Olkholovsky and Recami in 1992 [3], Landauer and Martin in 1994 [4], and Chiao and Steinberg in 1997 [5]). The fact that there is a time related to the tunnelling process has been observed experimentally [6-15]. However, the results of the experiments are ambiguous.

Many problems with time in quantum mechanics arise from the noncommutativity of the operators. The noncommutativity of the operators in quantum mechanics can be circumvented by using the concept of weak measurements. The concept of weak measurement was proposed by Aharonov, Albert, and Vaidman [16-21]. Such an approach has several advantages. It gives, in principle, the procedure for measuring the physical quantity. Second, since in the classical mechanics all quantities can have definite values simultaneously, weak measurements give the correct classical limit. The concept of weak measurements has been al- 
ready applied to the time problem in quantum mechanics [22-24].

Time in classical mechanics describes not a single state of the system but the process of the evolution. This property is an essential concept of time. We speak about the time belonging to a certain evolution of the system. If the measurement of the time disturbs the evolution we cannot attribute this measured duration to the undisturbed evolution. Therefore, we should require that the measurement of the time does not disturb the motion of the system. This means that the interaction of the system with the measuring device must be asymptotically weak. In quantum mechanics this means that we cannot use the strong measurements described by the von Neumann's projection postulate. We have to use the weak measurements of Aharonov, Albert, and Vaidman [16-21], instead.

We proceed as follows. In Section 2 we present the model of the weak measurements. Section 3 presents the time on condition that the system is in the given final state. In Section 4, our formalism is applied to the tunnelling time problem. In Section 5 the weak measurement of the quantum arrival time distribution is presented. Section 6 summarizes our findings.

\section{The concept of weak measurements}

In this section we present the concept of weak measurement, proposed by Aharonov, Albert, and Vaidman [16-21]. We measure the quantity represented by the operator $\widehat{A}$.

We have the detector in the initial state $|\Phi\rangle$. For a weak measurement to provide the meaningful information the measurements must be performed on an ensemble of identical systems. It is supposed that each system with its own detector is prepared in the same initial state. After measurement the readings of the detectors are collected and averaged.

Our model consists of the system $\mathbf{S}$ under consideration and of the detector D. The total Hamiltonian is

$$
\widehat{H}=\widehat{H}_{\mathrm{S}}+\widehat{H}_{\mathrm{D}}+\widehat{H}_{\mathrm{I}},
$$

where $\widehat{H}_{\mathrm{S}}$ and $\widehat{H}_{\mathrm{D}}$ are the Hamiltonians of the system and detector, respectively. We take the operator describing the interaction between the particle and the detector of the form [22, 23, 25-28]

$$
\widehat{H}_{I}=\lambda \widehat{q} \widehat{A},
$$

where $\lambda$ characterizes the strength of the interaction between the system and detector. The small parameter $\lambda$ ensures the undisturbance of the system's evolu- tion. The measurement duration is $\tau$. In this section we assume that the interaction strength $\lambda$ and the time $\tau$ are small. The operator $\widehat{q}$ acts in the Hilbert space of the detector. We require the spectrum of the operator $\widehat{q}$ to be continuous. For simplicity, we can consider this operator to be the coordinate of the detector. The momentum conjugate to $q$ is $p_{q}$.

The interaction operator (2) only slightly differs from the one used by Aharonov, Albert, and Vaidman [17]. The similar interaction operator has been considered by von Neumann [29] and has been widely used in the strong measurement models (e.g., [28, 30-34] and many others).

Hamiltonian (2) represents a constant force acting on the detector. This force results in the change of momentum of the detector. From the classical point of view, the change of the momentum is proportional to the force acting on the detector. Since interaction strength $\lambda$ and the duration of the measurement $\tau$ are small, the average $\langle\widehat{A}\rangle$ should not change significantly during the measurement. The action of the Hamiltonian (2) results in the small change of the mean detector momentum $\left\langle\widehat{p}_{q}\right\rangle-\left\langle\widehat{p}_{q}\right\rangle_{0}=-\lambda \tau\langle\widehat{A}\rangle$, where $\left\langle\widehat{p}_{q}\right\rangle_{0}=\left\langle\Phi(0)\left|\widehat{p}_{q}\right| \Phi(0)\right\rangle$ is the mean momentum of the detector at the beginning of the measurement and $\left\langle\widehat{p}_{q}\right\rangle=\left\langle\Phi(\tau)\left|\widehat{p}_{q}\right| \Phi(\tau)\right\rangle$ is the mean momentum of the detector after the measurement. Therefore, in analogy to [17], we define the "weak value" of the average $\langle\widehat{A}\rangle$,

$$
\langle\widehat{A}\rangle=\frac{\left\langle\widehat{p}_{q}\right\rangle_{0}-\left\langle\widehat{p}_{q}\right\rangle}{\lambda \tau} .
$$

At the moment $t=0$ the density matrix of the whole system is $\widehat{\rho}(0)=\widehat{\rho}_{\mathrm{S}}(0) \otimes \widehat{\rho}_{\mathrm{D}}(0)$, where $\widehat{\rho}_{\mathrm{S}}(0)$ is the density matrix of the system and $\widehat{\rho}_{\mathrm{D}}(0)=|\Phi\rangle\langle\Phi|$ is the density matrix of the detector. After the interaction the density matrix of the detector is $\widehat{\rho}_{\mathrm{D}}(t)=$ $\operatorname{Tr}_{S}\left\{\widehat{U}(t)\left(\widehat{\rho}_{\mathrm{S}}(0) \otimes|\Phi\rangle\langle\Phi|\right) \widehat{U}^{\dagger}(t)\right\}$, where $\widehat{U}(t)$ is the evolution operator. Later, for simplicity we shall neglect the Hamiltonian of the detector. Then, the evolution operator in the first-order approximation is [22]

$$
\widehat{U}(t) \approx \widehat{U}_{\mathrm{S}}(t)\left(1+\frac{\lambda \widehat{q}}{\mathrm{i} \hbar} \int_{0}^{t} \widetilde{A}\left(t_{1}\right) \mathrm{d} t_{1}\right)
$$

where $\widehat{U}_{\mathrm{S}}(t)$ is the evolution operator of the unperturbed system and $\widetilde{A}(t)=\widehat{U}_{\mathrm{S}}^{\dagger}(t) \widehat{A} \widehat{U}_{\mathrm{S}}(t)$. From Eq. (3) we obtain that the weak value coincides with the usual average $\langle\widehat{A}\rangle=\operatorname{Tr}\left\{\widehat{A} \widehat{\rho}_{\mathrm{S}}(0)\right\}$.

The influence of the weak measurement on the evolution of the measured system can be made arbitrary small using the small parameter $\lambda$. Therefore, after the 
interaction of the measured system with the detector we can try to measure the second observable $\widehat{B}$ using, as usual, the strong measurement. As far as our model gives the correct result for the value of $A$ averaged over the entire ensemble of the systems, now we can try to take the average only over the subensemble of the systems with the given value of the quantity $B$. We measure the momenta $p_{q}$ of each measuring device after the interaction with the system. Subsequently, we perform the final, postselection measurement of $B$ on the systems of our ensemble. Then we collect the outcomes $p_{q}$ only of the systems which have a given value of $B$.

The joint probability that the system has the given value of $B$ and the detector has the momentum $p_{q}(t)$ at the time moment $t$ is

$$
W\left(B, p_{q} ; t\right)=\operatorname{Tr}\left\{|B\rangle\left\langle B \mid p_{q}\right\rangle\left\langle p_{q}\right| \widehat{\rho}(t)\right\},
$$

where $\left|p_{q}\right\rangle$ is the eigenfunction of the momentum operator $\widehat{p}_{q}$. In quantum mechanics the probability that two quantities simultaneously have definite values does not always exist. If the joint probability does not exist then the concept of the conditional probability is meaningless. However, in our case operators $\widehat{B}$ and $\left|p_{q}\right\rangle\left\langle p_{q}\right|$ act in different spaces and commute, therefore, the probability $W\left(B, p_{q} ; t\right)$ exists.

Let us define the conditional probability, i.e. the probability that the momentum of the detector is $p_{q}$ provided that the system has the given value of $B$. This probability is given according to the Bayes theorem as

$$
W\left(p_{q} ; t \mid B\right)=\frac{W\left(B, p_{q} ; t\right)}{W(B ; t)},
$$

where $W(B ; t)=\operatorname{Tr}\{|B\rangle\langle B| \widehat{\rho}(t)\}$ is the probability that the system has the given value of $B$. The average momentum of the detector on condition that the system has the given value of $B$ is

$$
\left\langle p_{q}(t)\right\rangle=\int p_{q} W\left(p_{q} ; t \mid B\right) \mathrm{d} p_{q} .
$$

From Eqs. (3) and (6), in the first-order approximation we obtain the mean value of $A$ on condition that the system has the given value of $B$ (for analogy, see [22])

$$
\begin{aligned}
\langle A\rangle_{B}= & \frac{1}{2\left\langle B\left|\widehat{\rho}_{\mathrm{S}}\right| B\right\rangle}\langle\mid B\rangle\langle B|\widehat{A}+\widehat{A}| B\rangle\langle B \mid\rangle \\
& +\frac{1}{\mathrm{i} \hbar\left\langle B\left|\widehat{\rho}_{\mathrm{S}}\right| B\right\rangle} \\
& \quad \times\left(\langle q\rangle\left\langle p_{q}\right\rangle-\operatorname{Re}\left\langle\widehat{q}_{q}\right\rangle\right)\langle[|B\rangle\langle B|, \widehat{A}]\rangle .
\end{aligned}
$$

If the commutator $[|B\rangle\langle B|, \widehat{A}]$ in Eq. (7) is not zero then, even in the limit of the very weak measurement, the measured value depends on the particular detector. This fact means that in such a case we cannot obtain the definite value. Moreover, the coefficient $\left(\langle q\rangle\left\langle p_{q}\right\rangle-\operatorname{Re}\left\langle\widehat{q}_{q}\right\rangle\right)$ may be zero for the specific initial state of the detector, e. g., for the Gaussian distribution of the coordinate $q$ and momentum $p_{q}$.

\section{Definition of time under condition that the system is in the given final state}

The most-known problem related to time in quantum mechanics is the so-called "tunnelling time problem". We can raise another, more general, question about the time. Let us consider a system which evolves in time. Let $\chi$ is one of the observables of the system. During the evolution the value of $\chi$ changes. We are considering a subset $\Gamma$ of possible values of $\chi$. The question is how long the values of $\chi$ belong to this subset.

There is another version of the question. If we know the final state of the system, we may ask how long the values of $\chi$ belong to the subset under consideration when the system evolves from the initial to a final predefined state. The question about the tunnelling time belongs to such class of the problems. Really, in the tunnelling time problem we ask about the duration the particle spends in a specified region of the space, and we know that the particle has tunnelled out, i. e. it is on the other side of the barrier. We can expect that such a question can not always be answered. Here our goal is to obtain the conditions under which it is possible to answer such question.

One of the possibilities to solve the time problem is to answer what exactly the word "time" means. The meaning of every physical quantity is determined by the procedure of its measurement. Therefore, we have to construct a scheme of an experiment (this can be a gedanken experiment) which measures the quantity with the properties corresponding to the classical time.

\subsection{Model of the time measurement}

We consider a system evolving with time. Let one of the quantities describing the system be $\chi$. Operator $\hat{\chi}$ corresponds to this quantity. For simplicity we assume that the operator $\hat{\chi}$ has a continuous spectrum. The case with discrete spectrum will be considered later.

The measuring device interacts with the system only if $\chi$ is in some region near the point $\chi_{\mathrm{D}}$, the concrete value of which depends on the detector only. If we want 
to measure the time when the system is in a large region of $\chi$, one has to use many detectors. In the case of tunnelling a similar model was introduced by Steinberg [35] and developed in our paper [23]. The strong limit of such a model for analysis of the measurement effect for the quantum jumps has been used in [25].

We shall use the weak measurement concept described in Section 2. The operator $\widehat{A}$ will be represented by the operator

$$
\widehat{D}\left(\chi_{\mathrm{D}}\right)=\left|\chi_{\mathrm{D}}\right\rangle\left\langle\chi_{\mathrm{D}}\right|=\delta\left(\widehat{\chi}-\chi_{\mathrm{D}}\right) .
$$

It is assumed that after time $t$ the readings of the detectors are collected and averaged.

Hamiltonian (2) with $\widehat{A}$ given by Eq. (8) represents a constant force acting on the detector $\mathbf{D}$ when the quantity $\chi$ is very close to $\chi_{\mathrm{D}}$. This force induces the change of the detector's momentum. From the classical point of view, the change of the momentum is proportional to the time the particle spends in the region around $\chi_{\mathrm{D}}$, and the coefficient of proportionality equals the force acting on the detector. We assume that the change of the mean momentum of the detector is proportional to the time the constant force acts on the detector and that the time the particle spends in the detector's region coincides with the time the force acts on the detector.

We can replace the $\delta$-function by the narrow rectangle of height $1 / L$ and of width $L$ in the $\chi$ space. From Eq. (2) it follows that the force acting on the detector when the particle is in the region around $\chi_{\mathrm{D}}$ is $F=-\lambda / L$. The time the particle spends until time moment $t$ in the unit-length region is

$$
\tau(t)=-\frac{1}{\lambda}\left(\left\langle p_{q}(t)\right\rangle-\left\langle p_{q}\right\rangle\right),
$$

where $\left\langle p_{q}\right\rangle$ and $\left\langle p_{q}(t)\right\rangle$ are the mean initial momentum and the momentum after time $t$, respectively. If one wants to find the period the system spends in the region of the finite width, one must sum expressions of the type (9) many times.

When the operator $\hat{\chi}$ has a discrete spectrum, one may ask for how long the quantity $\chi$ has the value $\chi_{D}$. To answer this question the detector must interact with the system only when $\chi=\chi_{\mathrm{D}}$. If this is satisfied, the operator $\widehat{D}\left(\chi_{\mathrm{D}}\right)$ takes the form

$$
\widehat{D}\left(\chi_{\mathrm{D}}\right)=\left|\chi_{\mathrm{D}}\right\rangle\left\langle\chi_{\mathrm{D}}\right| .
$$

The force acting on the detector in this case equals to $-\lambda$. The duration that the quantity $\chi$ has the value $\chi_{\mathrm{D}}$ is given by Eq. (9), too. Note that now the formulae do not depend on the spectrum of the operator $\widehat{\chi}$.

\subsection{Dwell time}

To shorten the notation, the operator

$$
\widehat{F}\left(\chi_{\mathrm{D}}, t\right)=\int_{0}^{t} \widetilde{D}\left(\chi_{\mathrm{D}}, t_{1}\right) \mathrm{d} t_{1}
$$

is introduced, where

$$
\widetilde{D}\left(\chi_{\mathrm{D}}, t\right)=\widehat{U}_{\mathrm{S}}^{\dagger}(t) \widehat{D}\left(\chi_{\mathrm{D}}\right) \widehat{U}_{\mathrm{S}}(t) .
$$

After measurement, from the density matrix of the detector, in the first-order approximation we find that the average change of the detector momentum in the time interval $t$ is $-\lambda\left\langle\widehat{F}\left(\chi_{\mathrm{D}}, t\right)\right\rangle$. From Eq. (9) we obtain the dwell time until time moment $t$,

$$
\tau(\chi, t)=\langle\widehat{F}(\chi, t)\rangle .
$$

Then the time spent in the region $\Gamma$ is

$$
\tau(\Gamma ; t)=\int_{\Gamma} \tau(\chi, t) \mathrm{d} \chi=\int_{0}^{t} \mathrm{~d} t^{\prime} \int_{\Gamma} \mathrm{d} \chi P\left(\chi, t^{\prime}\right),
$$

where $P\left(\chi, t^{\prime}\right)=\langle\widetilde{D}(\chi, t)\rangle$ is the probability for the system to have the value $\chi$ at time moment $t^{\prime}$.

When $\chi$ is the coordinate of the particle, Eq. (14) yields the well-known expression for the dwell time $[3,23]$. This time is the average over the entire ensemble of the systems, regardless of their final states.

A relationship between dwell, transmission, and reflection times has recently been analysed in paper [36] while in paper [37] a relation between the group delay and the dwell time for quantum tunnelling is derived. It is shown that the group delay is equal to the dwell time plus self-interference delay which depends on the dispersion outside the barrier. The analysis shows that there is nothing superluminal in quantum tunnelling and the Hartman effect for tunnelling quantum particles can be explained by the saturation of the integrated probability density under the barrier.

\subsection{Definition of time under condition that the system is in a given final state}

Further, the case when the final state of the system is known will be considered. We may ask how long the values of $\chi$ belong to the subset under consideration, $\Gamma$, on condition that the system evolves to the definite final state $f$. More specifically, we might know that the final state of the system belongs to a certain subspace $\mathcal{H}_{f}$ of system's Hilbert space. The projection operator that projects the vectors from the Hilbert space of the system into the subspace $\mathcal{H}_{f}$ of the final states will be denoted $\widehat{P}_{f}$. As far as the considered model gives 
the correct result for the time averaged over the entire ensemble of the systems, we can try to take the average only over the subensemble of the systems with the given final states. At first, the momenta $p_{q}$ of each measuring device after the interaction with the system are measured. Subsequently, we perform the final, postselection measurement on the systems of the ensemble. Then we collect the outcomes $p_{q}$ only of those systems the final state of which turns out to belong to the subspace $\mathcal{H}_{f}$.

Using Eq. (7) in Section 2 we obtain the duration, on condition that the final state of the system belongs to the subspace $\mathcal{H}_{f}[22]$,

$$
\begin{aligned}
\tau_{f}(\chi, t)= & \frac{1}{2\left\langle\widetilde{P}_{f}(t)\right\rangle}\left\langle\widetilde{P}_{f}(t) \widehat{F}(\chi, t)+\widehat{F}(\chi, t) \widetilde{P}_{f}(t)\right\rangle \\
& +\frac{1}{\mathrm{i} \hbar\left\langle\widetilde{P}_{f}(t)\right\rangle}\left(\langle q\rangle\left\langle p_{q}\right\rangle-\operatorname{Re}\left\langle\widehat{q} \widehat{p}_{q}\right\rangle\right) \\
& \times\left\langle\left[\widetilde{P}_{f}(t), \widehat{F}(\chi, t)\right]\right\rangle .
\end{aligned}
$$

Equation (15) consists of two terms, thus, we can introduce two expressions with the dimension of time

$$
\tau_{f}^{(1)}(\chi, t)=\frac{1}{2\left\langle\widetilde{P}_{f}(t)\right\rangle}\left\langle\widetilde{P}_{f}(t) \widehat{F}(\chi, t)+\widehat{F}(\chi, t) \widetilde{P}_{f}(t)\right\rangle,
$$

$$
\tau_{f}^{(2)}(\chi, t)=\frac{1}{2 \mathrm{i}\left\langle\widetilde{P}_{f}(t)\right\rangle}\left\langle\left[\widetilde{P}_{f}(t), \widehat{F}(\chi, t)\right]\right\rangle .
$$

Then, the time the system spends in the subset $\Gamma$ on condition that the final state of the system belongs to the subspace $\mathcal{H}_{f}$ can be rewritten in the form

$$
\begin{aligned}
& \tau_{f}(\chi, t) \\
& \quad=\tau_{f}^{(1)}(\chi, t)+\frac{2}{\hbar}\left(\langle q\rangle\left\langle p_{q}\right\rangle-\operatorname{Re}\left\langle\widehat{q}_{q}\right\rangle\right) \tau_{f}^{(2)}(\chi, t) .
\end{aligned}
$$

The quantities $\tau_{f}^{(1)}(\chi, t)$ and $\tau_{f}^{(2)}(\chi, t)$ are related to the real and imaginary parts of the complex time introduced by Sokolovski et al. [38]. In our model the quantity $\tau_{f}(\chi, t)$ is real, contrary to the complex-time approach. The components of time $\tau_{f}^{(1)}$ and $\tau_{f}^{(2)}$ are real, too. Therefore, this time can be interpreted as the duration of an event.

If the commutator $\left[\widetilde{P}_{f}(t), \widehat{F}(\chi, t)\right]$ in Eq. (15) is not zero then, even in the limit of very weak measurements, the measured value depends on the particular detector used. This means that in such a case we cannot obtain a definite value for the conditional time. Moreover, the coefficient $\left(\langle q\rangle\left\langle p_{q}\right\rangle-\operatorname{Re}\left\langle\widehat{q} \widehat{p}_{q}\right\rangle\right)$ can be zero for the specific initial state of the detector, e. g., for the Gaussian distribution of the coordinate $q$ and momentum $p_{q}$.

The conditions to determine the time uniquely in a case when the final state of the system is known takes, thus, the form

$$
\left[\widetilde{P}_{f}(t), \widehat{F}(\chi, t)\right]=0,
$$

which can be understood from the general principles of quantum mechanics, too. Now, we ask how long the values of $\chi$ belong to a certain subset when the system evolves to the given final state under assumption that the final state of the system is known with certainty. In addition, we want to have some information about the values of the quantity $\chi$. However, if the final state is known with certainty, we may not know the values of $\chi$ in the past and, vice versa, if we know something about $\chi$, we may not definitely determine the final state. Therefore, in such a case the question about the time when the system evolves to the given final state cannot be answered definitely and the conditional time has no reasonable meaning.

The quantity $\tau_{f}(t)$ according to Eqs. (15) and (16) has many properties of the classical time. So, if the final states $\{f\}$ constitute the full set, then the corresponding projection operators obey the equality of completeness $\sum_{f} \widehat{P}_{f}=1$. Then, from Eq. (15) we obtain the expression

$$
\sum_{f}\left\langle\widetilde{P}_{f}(t)\right\rangle \tau_{f}(\chi, t)=\tau(\chi, t) .
$$

The quantity $\left\langle\widetilde{P}_{f}(t)\right\rangle$ is the probability that the system at the time $t$ is in the state $f$. Equation (20) shows that the full duration equals the average over all possible final states, as it is a case in the classical physics. From Eq. (20) and Eqs. (16), (17) it follows

$$
\begin{aligned}
& \sum_{f}\left\langle\widetilde{P}_{f}(t)\right\rangle \tau_{f}^{(1)}(\chi, t)=\tau(\chi, t), \\
& \sum_{f}\left\langle\widetilde{P}_{f}(t)\right\rangle \tau_{f}^{(2)}(\chi, t)=0 .
\end{aligned}
$$

We suppose that the quantities $\tau_{f}^{(1)}(\chi, t)$ and $\tau_{f}^{(2)}(\chi, t)$ can be useful even in the case when the time has no definite value, since in the tunnelling time problem the quantities (16) and (17) correspond to real and imaginary parts of the complex time, respectively [23].

The eigenfunctions of the operator $\hat{\chi}$ constitute the full set $\int|\chi\rangle\langle\chi| \mathrm{d} \chi=1$, where the integral must be replaced by the sum for the discrete spectrum of the 
operator $\widehat{\chi}$. From Eqs. (8), (11), (15) we obtain the equality

$$
\int \tau_{f}(\chi, t) \mathrm{d} \chi=t
$$

which shows that the time during which the quantity $\chi$ has any value equals to $t$, as it is in the classical physics.

\subsection{Example: Two-level system}

The obtained formalism can be applied to the tunnelling time problem [23]. In this section, however, we will consider a simpler system than the tunnelling particle, i. e. a two-level system. The system is forced by the perturbation $V$ that causes the jumps from one state to another. The time the system is in a given state will be calculated.

The Hamiltonian of this system is

$$
\widehat{H}=\widehat{H}_{0}+\widehat{V},
$$

where $\widehat{H}_{0}=\hbar \omega \widehat{\sigma}_{3} / 2$ is the Hamiltonian of the unperturbed system and $\widehat{V}=v \widehat{\sigma}_{+}+v^{*} \widehat{\sigma}_{-}$is the perturbation. Here $\widehat{\sigma}_{1}, \widehat{\sigma}_{2}, \widehat{\sigma}_{3}$ are Pauli matrices and $\sigma_{ \pm}=$ $\frac{1}{2}\left(\widehat{\sigma}_{1} \pm \mathrm{i} \widehat{\sigma}_{2}\right)$. The Hamiltonian $\widehat{H}_{0}$ has two eigenfunctions $|0\rangle$ and $|1\rangle$ with the eigenvalues $-\hbar \omega / 2$ and $\hbar \omega / 2$, respectively. The initial state of the system assumed to be $|0\rangle$.

From Eq. (13) we obtain the times the system spends in the energy levels 0 and 1 , respectively,

$$
\begin{aligned}
& \tau(0, t)=\frac{1}{2}\left(1+\frac{\omega^{2}}{\Omega^{2}}\right) t+\frac{1}{2 \Omega} \sin (\Omega t)\left(1-\frac{\omega^{2}}{\Omega^{2}}\right) \\
& \tau(1, t)=\frac{1}{2}\left(1-\frac{\omega^{2}}{\Omega^{2}}\right) t-\frac{1}{2 \Omega} \sin (\Omega t)\left(1-\frac{\omega^{2}}{\Omega^{2}}\right)
\end{aligned}
$$

where $\Omega=\sqrt{\omega^{2}+4(|v| / \hbar)^{2}}$. From Eqs. (16) and (17) we can obtain the conditional time. The components (16) and (17) of the time the system spends in the level 0 under condition that the final state after measurement is $|1\rangle$ are

$$
\begin{aligned}
\tau_{1}^{(1)}(0, t) & =\frac{t}{2} \\
\tau_{1}^{(2)}(0, t) & =\frac{\omega}{2 \Omega}\left[1-t \cot \left(\frac{\Omega}{2} t\right)\right] .
\end{aligned}
$$

When $\Omega t=2 \pi n$, where $n \in \mathbb{Z}$, the quantity $\tau_{1}^{(2)}(0, t)$ tends to infinity. This happens because at these time moments the system is in the state $|1\rangle$ with the probability 0 , and one cannot consider the interaction with the detector as very weak.

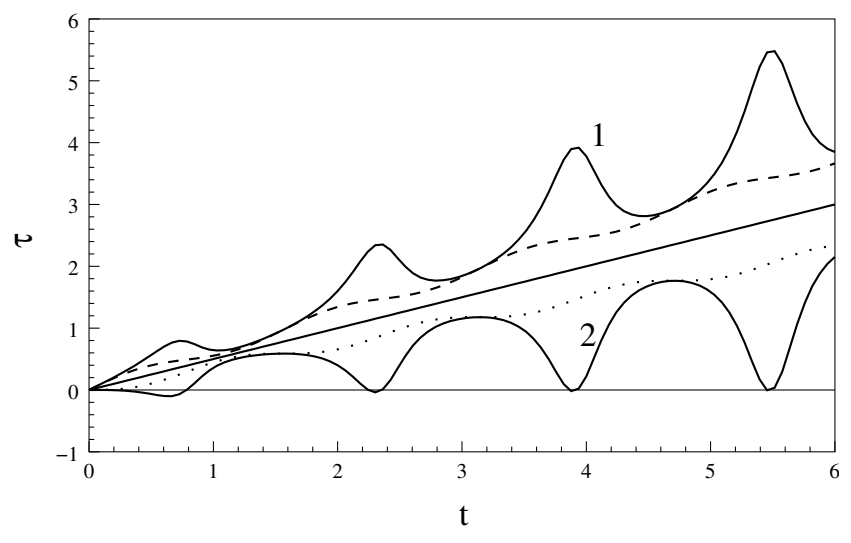

Fig. 1. The time the system spends in the energy level $0, \tau(0, t)$ (dashed line), and level 1, $\tau(1, t)$ (dotted line), according to Eqs. (25) and (26), respectively. The quantity $\tau_{1}^{(1)}(0, t)$, Eq. (27), is shown as solid straight line. The quantities $\tau_{0}^{(1)}(0, t)$ and $\tau_{0}^{(1)}(1, t)$, shown by curves 1 and 2 , were calculated according to Eqs. (30) and (31), respectively. The parameters are $\omega=2, \Omega=4$.

On the other hand, the components of the time the system spends in level 0 under condition that the final state is $|0\rangle$ are

$\tau_{0}^{(1)}(0, t)$

$$
=\frac{\left(1+3 \frac{\omega^{2}}{\Omega^{2}}\right) t+\left(1-\frac{\omega^{2}}{\Omega^{2}}\right)\left(\frac{2}{\Omega} \sin (\Omega t)+t \cos (\Omega t)\right)}{2\left[\left(1+\frac{\omega^{2}}{\Omega^{2}}\right)+\left(1-\frac{\omega^{2}}{\Omega^{2}}\right) \cos (\Omega t)\right]},
$$

$\tau_{0}^{(2)}(0, t)$

$$
=\frac{\frac{\omega}{\Omega}\left(1-\frac{\omega^{2}}{\Omega^{2}}\right) \sin \left(\frac{\Omega}{2} t\right)\left[t \cos \left(\frac{\Omega}{2} t\right)-\frac{2}{\Omega} \sin \left(\frac{\Omega}{2} t\right)\right]}{2\left[\left(1+\frac{\omega^{2}}{\Omega^{2}}\right)+\left(1-\frac{\omega^{2}}{\Omega^{2}}\right) \cos (\Omega t)\right]} .
$$

The time the system spends in level 1 under condition that the final state is $|0\rangle$ can be expressed as

$$
\tau_{0}^{(1)}(1, t)=\frac{\left(1-\frac{\omega^{2}}{\Omega^{2}}\right)\left(t+t \cos (\Omega t)-\frac{2}{\Omega} \sin (\Omega t)\right)}{2\left[\left(1+\frac{\omega^{2}}{\Omega^{2}}\right)+\left(1-\frac{\omega^{2}}{\Omega^{2}}\right) \cos (\Omega t)\right]} .
$$

The quantities $\tau(0, t), \tau(1, t), \tau_{1}^{(1)}(0, t), \tau_{0}^{(1)}(0, t)$, and $\tau_{0}^{(1)}(1, t)$ are shown in Fig. 1. The quantity $\tau_{0}^{(2)}(0, t)$ is shown in Fig. 2. Note that the partial durations at the given final state are not necessarily monotonic as it is with the full duration, because the final state at different time moments can be reached by different paths. We can interpret the quantity $\tau_{0}^{(1)}(0, t)$ as the time the system spends in the level 0 on condition that the final state is $|0\rangle$, but at certain time moments 


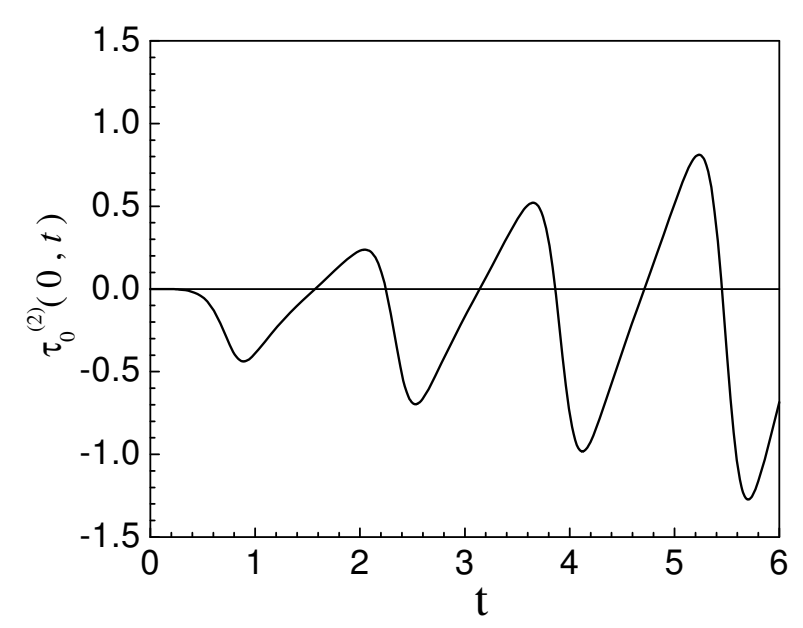

Fig. 2. The quantity $\tau_{0}^{(2)}(0, t)$, Eq. (30). The parameters are the same as in Fig. 1.

this quantity is greater than $t$. In such cases the quantity $\tau_{0}^{(1)}(1, t)$ becomes negative at certain times. This is a consequence of the fact that for the system under consideration the condition (19) is not fulfilled. The peculiarities of the behaviour of the conditional times show that it is impossible to decompose the unconditional time into two components having all classical properties of the time.

\section{Tunnelling time}

The best-known problem of time in quantum mechanics is the so-called "tunnelling time problem". This problem is still the subject of much controversy, since numerous theories contradict each other in their predictions for the "tunnelling time". Many of the theoretical approaches can be divided into three categories. First, one can study the evolution of the wave packets through the barrier and get the phase time. However, the correctness of the definition of this time is highly questionable [39]. Another approach is based on the determination of a set of dynamic paths, i. e. the calculation of the time the different paths spend in the barrier and averaging over the set of the paths. The paths can be found from the Feynman path integral formalism [38], from the Bohm approach [40-45], or from the Wigner distribution [46]. The third class uses a physical clock which is used for determination of the time elapsed during the tunnelling (Büttiker and Landauer used an oscillatory barrier [39], Baz' suggested the Larmor time [47]). One more approach is based on a model for tunnelling based on stochastic interpretation of quantum mechanics [48-51].
The problems arise also from the fact that the arrival time of a particle to a definite spatial point is a classical concept. Its quantum counterpart is problematic even for the free particle case. In classical mechanics, for the determination of the time the particle spends moving along a certain trajectory, one has to measure the position of the particle at two different moments of time. In quantum mechanics this procedure does not work. From Heisenberg's uncertainty principle it follows that we cannot measure the position of a particle without alteration of its momentum. To determine exactly the arrival time of a particle, one has to measure the position of the particle with great precision. Because of the measurement, the momentum of the particle will have a big uncertainty and the second measurement will be indefinite. If we want to ask about the time in quantum mechanics, we need to define the procedure of measurement. We can measure the position of the particle only with a finite precision and get a distribution of the possible positions. Applying such a measurement, we can expect to obtain not a single value of the traversal time but a distribution of times.

In the paper [52] the tunnelling time distribution for photon tunnelling is analysed theoretically as a spacetime correlation phenomenon between the emission and absorption of a photon on the two sides of a barrier. The analysis is based on an appropriate counting rate formula derived at first order in the photon-detector interaction and used in treating space-time correlations between photons.

There are two different but related questions connected with the tunnelling time problem [53]:

(i) How much time does the tunnelling particle spend under the barrier?

(ii) At what time does the particle arrive at the point behind the barrier?

There have been many attempts to answer these questions. However, there are several papers showing that according to quantum mechanics the question (i) makes no sense [53-56]. Our goal is to investigate the possibility to determine the tunnelling time using weak measurements.

\subsection{Determination of the tunnelling time}

To answer the question of how much time does the tunnelling particle spend under the barrier, we need a criterion of the tunnelling. The following criterion is accepted: the particle had tunnelled in the case when 
it was in front of the barrier at first and later it was found behind the barrier. We shall require that the mean energy of the particle and the energy uncertainty should be less than the height of the barrier. Following this criterion, the operator corresponding to the "tunnellingflag" observable is introduced

$$
\widehat{f}_{T}(X)=\Theta(\widehat{x}-X),
$$

where $\Theta$ is the Heaviside unit step function and $X$ is a point behind the barrier. This operator projects the wave function onto the subspace of functions localized behind the barrier. The operator has two eigenvalues: 0 and 1 . The eigenvalue 0 corresponds to the fact that the particle has not tunnelled out, while the eigenvalue 1 corresponds to the appearance of particle behind the barrier.

We will work with the Heisenberg representation. In this representation, the tunnelling-flag operator becomes

$$
\widetilde{f}_{T}(t, X)=\exp \left(\frac{\mathrm{i}}{\hbar} \widehat{H} t\right) \widehat{f}_{T}(X) \exp \left(-\frac{\mathrm{i}}{\hbar} \widehat{H} t\right) .
$$

To take into account all the tunnelled particles, the limit $t \rightarrow+\infty$ must be taken. So, the "tunnelling-flag" observable in the Heisenberg picture is represented by the operator $\widetilde{f}_{T}(\infty, X)=\lim _{t \rightarrow+\infty} \widetilde{f}_{T}(t, X)$. One can obtain the explicit expression for this operator.

The operator $\widetilde{f}_{T}(t, X)$ obeys the standard equation

$$
\mathrm{i} \hbar \frac{\partial}{\partial t} \tilde{f}_{T}(t, X)=\left[\tilde{f}_{T}(t, X), \widehat{H}\right] .
$$

The commutator in Eq. (34) can be expressed as

$$
\begin{aligned}
& {\left[\widetilde{f}_{T}(t, X), \widehat{H}\right]} \\
& \quad=\exp \left(\frac{\mathrm{i}}{\hbar} \widehat{H} t\right)\left[\widehat{f}_{T}(X), \widehat{H}\right] \exp \left(-\frac{\mathrm{i}}{\hbar} \widehat{H} t\right) .
\end{aligned}
$$

If the Hamiltonian has the form

$$
\widehat{H}=\frac{1}{2 M} \widehat{p}^{2}+V(\widehat{x}),
$$

then the commutator becomes

$$
\left[\widehat{f}_{T}(X), \widehat{H}\right]=\mathrm{i} \hbar \widehat{J}(X),
$$

where $\widehat{J}(X)$ is the probability flux operator,

$$
\widehat{J}(x)=\frac{1}{2 M}(|x\rangle\langle x|\widehat{p}+\widehat{p}| x\rangle\langle x|) .
$$

Therefore, the following equation for the commutator can be written:

$$
\left[\widetilde{f}_{T}(t, X), \widehat{H}\right]=\mathrm{i} \hbar \widetilde{J}(X, t) .
$$

The initial condition for the function $\widetilde{f}(t, X)$ can be defined as

$$
\widetilde{f}_{T}(t=0, X)=\widehat{f}_{T}(X) .
$$

From Eqs. (34) and (37) we obtain the equation for the evolution of the tunnelling-flag operator

$$
\mathrm{i} \hbar \frac{\partial}{\partial t} \widetilde{f}_{T}(t, X)=\mathrm{i} \hbar \widetilde{J}(X, t) .
$$

From Eq. (38) and the initial condition, an explicit expression for the tunnelling-flag operator follows:

$$
\widetilde{f}_{T}(t, X)=\widehat{f}_{T}(X)+\int_{0}^{t} \widetilde{J}\left(X, t_{1}\right) \mathrm{d} t_{1} .
$$

In the already mentioned question of how much time does the tunnelling particle spend under the barrier, we shall be interested in those particles, which we know with certainty have tunnelled out. In addition, we want to have some information about the location of the particle. However, one may ask whether the quantum mechanics allows one to have the information about the tunnelling and location simultaneously? The projection operator

$$
\widehat{D}(\Gamma)=\int_{\Gamma}|x\rangle\langle x| \mathrm{d} x
$$

represents the probability for the particle to be in the region $\Gamma$. Here $|x\rangle$ is the eigenfunction of the coordinate operator. In the Heisenberg representation this operator takes the form

$$
\widetilde{D}(\Gamma, t)=\exp \left(\frac{\mathrm{i}}{\hbar} \widehat{H} t\right) \widehat{D}(\Gamma) \exp \left(-\frac{\mathrm{i}}{\hbar} \widehat{H} t\right) .
$$

From Eqs. (36), (39), and (41) we see that the operators $\widetilde{D}(\Gamma, t)$ and $\widetilde{f}_{T}(\infty, X)$ in general do not commute. This means that we cannot simultaneously have the information about the tunnelling and location of the particle. If we know with certainty that the particle has tunnelled out then we can say nothing about its location in the past, and if we know something about the location of the particle, we cannot determine definitely whether the particle has tunnelled out. Therefore, the question of how much time does the tunnelling particle spend under the barrier cannot have definite answer, if the question is so posed that its precise definition requires the existence of the joint probability that the particle is found in $\Gamma$ at time $t$ and whether or not it is found on the right side of the barrier at a sufficiently later time. A similar analysis has been performed in [56]. It has been shown that, due to noncommutativity of operators, there exists no unique decomposition of the dwell time. 


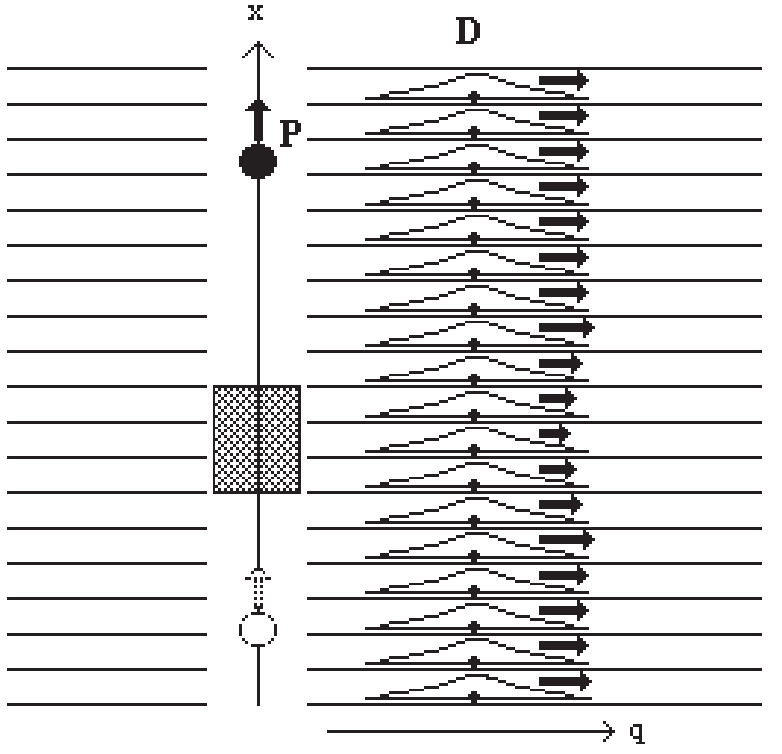

Fig. 3. The configuration of the measurement of the tunnelling time. The particle $\mathbf{P}$ is tunnelling along $x$ coordinate and weakly interacts with detectors $\mathbf{D}$. The barrier is represented by the hatched rectangle. The interaction with the individual detectors occurs only in the narrow region limited by the horizontal lines. The changes of the momenta of the detectors are represented by arrows.

This conclusion is, however, not negative altogether. We know that $\int_{-\infty}^{+\infty}|x\rangle\langle x| \mathrm{d} x=1$ and $\left[1, \widetilde{f}_{T}(\infty, X)\right]=0$. Therefore, if the region $\Gamma$ is large enough, one has a possibility to answer the question about the tunnelling time.

From the fact that the operators $\widetilde{D}(\Gamma, t)$ and $\widetilde{f}_{T}(\infty, X)$ do not commute we can predict that the measurement of the tunnelling time will yield a value dependent on the particular detection scheme. We shall assume the detector is made so that it yields some value. But if we try to measure noncommuting observables, the measured values depend on the interaction between the detector and the measured system. So, in the definition of the Larmor time there is a dependence on the type of boundary attributed to the magnetic-field region [3].

\subsection{Model of the time measurement}

We consider a model for the tunnelling time measurement which is somewhat similar to the gedanken experiment used to obtain the Larmor time but is simpler and more transparent. This model was proposed by Steinberg [35], however, it was treated in a nonstandard way, introducing complex probabilities. Here we shall use only the formalism of the standard quantum mechanics.
Our system consists of a particle $\mathbf{P}$ and a number of detectors D [23]. Each detector interacts with the particle only in a narrow region of space. The configuration of the system is shown in Fig. 3. When the interaction of the particle with the detectors is weak, the detectors do not influence the state of the particle. Therefore, we can analyse the action of the detectors separately. This model is a particular case of time measurement presented in Section 3.1, with $\chi_{\mathrm{D}}$ being the position of the detector $x_{\mathrm{D}}$. Similar calculations were done for detector's position rather than momentum by Iannaccone [57].

At the moment $t=0$ the particle is before the barrier, therefore, $\left\langle x\left|\rho_{\mathrm{P}}(0)\right| x^{\prime}\right\rangle \neq 0$ only when $x<0$ and $x^{\prime}<0$, where $\widehat{\rho}_{\mathrm{P}}(0)$ is the density matrix of the particle $\mathbf{P}$.

\subsection{Measurement of the dwell time}

As in Section 3.2 we obtain the time the particle spends in the unit length region between time instances 0 and $t$ :

$$
\tau^{\mathrm{Dw}}(x, t)=\langle\widehat{F}(x, t)\rangle .
$$

The time spent in the space region restricted by the coordinates $x_{1}$ and $x_{2}$ is

$$
\begin{aligned}
t^{\mathrm{Dw}}\left(x_{2}, x_{1}\right) & =\int_{x_{1}}^{x_{2}} \tau^{\mathrm{Dw}}(x, t \rightarrow \infty) \mathrm{d} x \\
& =\int_{x_{1}}^{x_{2}} \mathrm{~d} x \int_{0}^{\infty} \rho(x, t) \mathrm{d} t
\end{aligned}
$$

which is a well-known expression for the dwell time [3]: the dwell time is the average over an entire ensemble of particles regardless whether they tunnelled or not. The expression for the dwell time obtained in our model is the same as the well-known expression obtained by other authors. Therefore, we can expect that our model can yield a reasonable expression for the tunnelling time as well.

\subsection{Conditional probabilities and the tunnelling time}

Having seen that our model is capable to give the time averaged over entire ensemble of the particles, let us now take the average over the subensemble of the tunnelled particles only. This will be done similarly to Section 3.3 with $\widehat{P}_{f}$ replaced by the tunnelling-flag operator $\widehat{f}_{T}(X)$ defined by Eq. (32). From Eq. (15) we 
obtain the duration the tunnelled particle spends in the unit length region around $x$ until time $t$ [23]

$$
\begin{aligned}
\tau(x, t)= & \frac{1}{2\left\langle\widetilde{f}_{T}(t, X)\right\rangle} \\
& \times\left\langle\widetilde{f}_{T}(t, X) \widehat{F}(x, t)+\widehat{F}(x, t) \widetilde{f}_{T}(t, X)\right\rangle \\
& +\frac{1}{i \hbar\left\langle\widetilde{f}_{T}(t, X)\right\rangle}\left(\langle q\rangle\left\langle p_{q}\right\rangle-\operatorname{Re}\left\langle\widehat{q}_{q}\right\rangle\right) \\
& \times\left\langle\left[\widetilde{f}_{T}(t, X), \widehat{F}(x, t)\right]\right\rangle .
\end{aligned}
$$

The obtained expression (44) for the tunnelling time is real, contrary to the complex-time approach. It should be noted that this expression, even in the limit of the weak measurement, depends on a particular detector. If the commutator $\left[\widetilde{f}_{T}(t, X), \widehat{F}(x, t)\right]$ is zero, the time has a well-defined value. If the commutator is not zero, only the integral of this expression over a large region has meaning of an asymptotic time related to the large region as we will see in Section 4.7.

Equation (44) can be rewritten as a sum of two terms, the first term being independent and the second dependent on the detector, i. e.

$$
\begin{aligned}
& \tau(x, t) \\
& \quad=\tau^{\operatorname{Tun}}(x, t)+\frac{2}{\hbar}\left(\langle q\rangle\left\langle p_{q}\right\rangle-\operatorname{Re}\left\langle\widehat{q} \widehat{p}_{q}\right\rangle\right) \tau_{\text {corr }}^{\text {Tun }}(x, t),
\end{aligned}
$$

where

$$
\begin{aligned}
\tau^{\text {Tun }}(x, t)= & \frac{1}{2\left\langle\widetilde{f}_{T}(t, X)\right\rangle} \\
& \times\left\langle\widetilde{f}_{T}(t, X) \widehat{F}(x, t)+\widehat{F}(x, t) \widetilde{f}_{T}(t, X)\right\rangle,
\end{aligned}
$$$$
\tau_{\text {corr }}^{\operatorname{Tun}}(x, t)=\frac{1}{2 \mathrm{i}\left\langle\widetilde{f}_{T}(t, X)\right\rangle}\left\langle\left[\widetilde{f}_{T}(t, X), \widehat{F}(x, t)\right]\right\rangle .
$$

The quantities $\tau^{\text {Tun }}(x, t)$ and $\tau_{\text {corr }}^{\text {Tun }}(x, t)$ are independent of the detector.

In order to separate the tunnelled and reflected particles, the limit $t \rightarrow \infty$ should be taken. Otherwise, the particles that tunnelled after the time $t$ will not contribute. If we introduce the operators

$$
\begin{aligned}
& \widehat{F}(x)=\int_{0}^{\infty} \widetilde{D}\left(x, t_{1}\right) \mathrm{d} t_{1}, \\
& \widehat{N}(x)=\int_{0}^{\infty} \widetilde{J}\left(x, t_{1}\right) \mathrm{d} t_{1},
\end{aligned}
$$

then from Eq. (39) it follows that the operator $\widetilde{f}_{T}(\infty, X)$ is $\widehat{f}_{T}(X)+\widehat{N}(X)$. If the particle is initially before the barrier, then

$$
\widehat{f}_{T}(X) \widehat{\rho}_{P}(0)=\widehat{\rho}_{P}(0) \widehat{f}_{T}(X)=0 .
$$

In the limit $t \rightarrow \infty$ the tunnelling times become

$$
\begin{aligned}
\tau^{\operatorname{Tun}}(x) & =\frac{1}{2\langle\widehat{N}(X)\rangle}\langle\widehat{N}(X) \widehat{F}(x)+\widehat{F}(x) \widehat{N}(X)\rangle, \\
\tau_{\text {corr }}^{\operatorname{Tun}}(x) & =\frac{1}{2 \mathrm{i}\langle\widehat{N}(X)\rangle}\langle[\widehat{N}(X), \widehat{F}(x)]\rangle .
\end{aligned}
$$

Let us define an "asymptotic time" as the integral of $\tau(x, \infty)$ over a wide region containing the barrier. Since the integral of $\tau_{\text {corr }}^{\text {Tun }}(x)$ is very small compared to that of $\tau^{\text {Tun }}(x)$ as we shall see later, the asymptotic time is effectively the integral of $\tau^{\text {Tun }}(x)$ only. This allows us to identify $\tau^{\text {Tun }}(x)$ as the "density of the tunnelling time".

In many cases for the simplification of mathematics it is common to write the integrals over time as the integrals from $-\infty$ to $+\infty$. In our model we cannot, without additional assumptions, integrate Eqs. (48), (49) from $-\infty$ because the negative time means the motion of the particle to the initial position. If some particle in the initial wave packet had a negative momentum then in the limit $t \rightarrow-\infty$ it was behind the barrier and contributed to the tunnelling time.

\subsection{Properties of the tunnelling time}

As stated, the question of how much time does a tunnelling particle spend under the barrier has no exact answer. We can determine only the time the tunnelling particle spends in a large region containing the barrier. In our model this time is expressed as an integral of quantity (50) over this region. In order to determine the properties of this integral it is useful to determine the properties of the integrand.

To be able to expand the range of integration over time to $-\infty$, it is necessary to have the initial wave packet far to the left from the points under the investigation and this wave packet must consist only of the waves moving in the positive direction.

It is convenient to perform calculations in the energy representation. Eigenfunctions of the Hamiltonian $\widehat{H}_{\mathrm{P}}$ 
are $|E, \alpha\rangle$, where $\alpha= \pm 1$. The sign + or - corresponds to the positive or negative initial direction of the wave, respectively. Outside the barrier these eigenfunctions are

$$
\langle x \mid E,+\rangle=\left\{\begin{array}{l}
\sqrt{\frac{M}{2 \pi \hbar p_{E}}}\left[\exp \left(\frac{\mathrm{i}}{\hbar} p_{E} x\right)\right. \\
\left.+r(E) \exp \left(-\frac{\mathrm{i}}{\hbar} p_{E} x\right)\right] \\
x<0, \\
\sqrt{\frac{M}{2 \pi \hbar p_{E}}} t(E) \exp \left(\frac{\mathrm{i}}{\hbar} p_{E} x\right), \\
\quad x>L,
\end{array}\right.
$$

where $t(E)$ and $r(E)$ are transmission and reflection amplitudes, respectively, and

$$
p_{E}=\sqrt{2 M E} .
$$

Here $M$ is the mass of the particle. The barrier is in the region between $x=0$ and $x=L$. These eigenfunctions are orthonormal, i. e.

$$
\left\langle E, \alpha \mid E^{\prime}, \alpha^{\prime}\right\rangle=\delta_{\alpha, \alpha^{\prime}} \delta\left(E-E^{\prime}\right) .
$$

The evolution operator is

$$
\widehat{U}_{\mathrm{P}}(t)=\sum_{\alpha} \int_{0}^{\infty}|E, \alpha\rangle\langle E, \alpha| \exp \left(-\frac{\mathrm{i}}{\hbar} E t\right) \mathrm{d} E .
$$

Then the operator $\widehat{F}(x)$ assumes the form

$$
\begin{aligned}
\widehat{F}(x)= & \int_{-\infty}^{\infty} \mathrm{d} t_{1} \sum_{\alpha, \alpha^{\prime}} \iint \mathrm{d} E \mathrm{~d} E^{\prime}|E, \alpha\rangle\langle E, \alpha \mid x\rangle \\
& \times\left\langle x \mid E^{\prime}, \alpha^{\prime}\right\rangle\left\langle E^{\prime}, \alpha^{\prime}\right| \exp \left[\frac{\mathrm{i}}{\hbar}\left(E-E^{\prime}\right) t_{1}\right],
\end{aligned}
$$

where the integral over the time yields $2 \pi \hbar \delta\left(E-E^{\prime}\right)$, and therefore,

$$
\begin{aligned}
& \widehat{F}(x) \\
& \quad=2 \pi \hbar \sum_{\alpha, \alpha^{\prime}} \int \mathrm{d} E|E, \alpha\rangle\langle E, \alpha \mid x\rangle\left\langle x \mid E, \alpha^{\prime}\right\rangle\left\langle E, \alpha^{\prime}\right| .
\end{aligned}
$$

Similarly, we find

$$
\begin{aligned}
& \widehat{N}(x) \\
& \quad=2 \pi \hbar \sum_{\alpha, \alpha^{\prime}} \int \mathrm{d} E|E, \alpha\rangle\left\langle E, \alpha|\widehat{J}(x)| E, \alpha^{\prime}\right\rangle\left\langle E, \alpha^{\prime}\right| .
\end{aligned}
$$

If the initial wave packet consisting only of the waves moving in the positive direction is assumed, then one has

$$
\begin{aligned}
& \langle\widehat{N}(x)\rangle \\
& =2 \pi \hbar \int \mathrm{d} E\langle\mid E,+\rangle\langle E,+|\widehat{J}(x)| E,+\rangle\langle E,+\mid\rangle, \\
& \langle\widehat{F}(x) \widehat{N}(X)\rangle \\
& =4 \pi^{2} \hbar^{2} \sum_{\alpha} \int \mathrm{d} E\langle\mid E,+\rangle\langle E,+\mid x\rangle\langle x \mid E, \alpha\rangle \\
& \quad \times\langle E, \alpha|\widehat{J}(X)| E,+\rangle\langle E,+\mid\rangle .
\end{aligned}
$$

From the condition $X>L$ it follows that

$$
\langle\widehat{N}(X)\rangle=\int \mathrm{d} E\langle\mid E,+\rangle|t(E)|^{2}\langle E,+\mid\rangle .
$$

For $x<0$ we obtain the following expressions for the quantities $\tau^{\text {Tun }}(x, t)$ and $\tau_{\text {corr }}^{\text {Tun }}(x, t)$ :

$$
\begin{aligned}
\tau^{\text {Tun }}(x, t)= & \frac{M}{\langle\widehat{N}(X)\rangle} \int \mathrm{d} E\langle\mid E,+\rangle \frac{1}{2 p_{E}}|t(E)|^{2} \\
& \times\left[2+r(E) \exp \left(-2 \frac{\mathrm{i}}{\hbar} p_{E} x\right)\right. \\
& \left.+r^{*}(E) \exp \left(2 \frac{\mathrm{i}}{\hbar} p_{E} x\right)\right]\langle E,+\mid\rangle,
\end{aligned}
$$

$$
\begin{aligned}
\tau_{\text {corr }}^{\text {Tun }}(x, t)= & \frac{M}{2\langle\widehat{N}(X)\rangle} \int \mathrm{d} E\langle\mid E,+\rangle \frac{1}{\mathrm{i} p_{E}}|t(E)|^{2} \\
& \times\left[r(E) \exp \left(-2 \frac{\mathrm{i}}{\hbar} p_{E} x\right)\right. \\
& \left.-r^{*}(E) \exp \left(2 \frac{\mathrm{i}}{\hbar} p_{E} x\right)\right]\langle E,+\mid\rangle .
\end{aligned}
$$




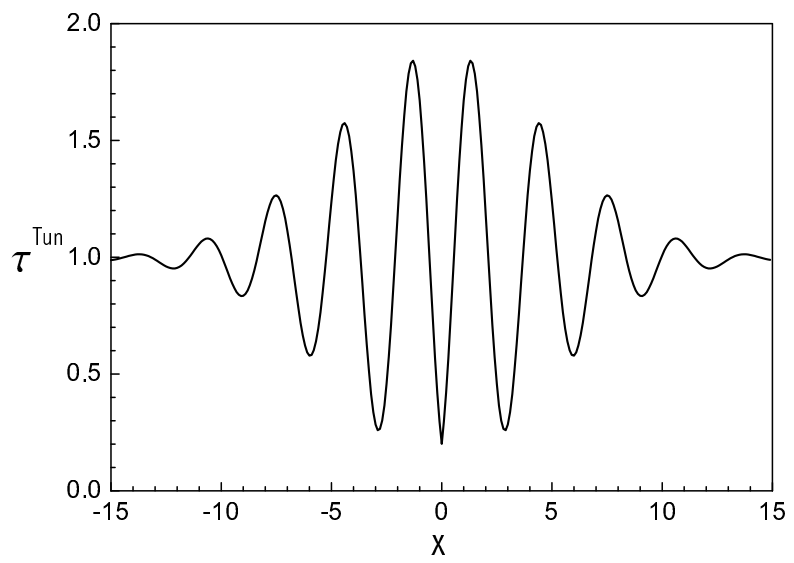

Fig. 4. Asymptotic time density for a $\delta$-function barrier with the parameter $\Omega=2$. The barrier is located at the point $x=0$. The units are such that $\hbar=1, M=1$, and the average momentum of the Gaussian wave packet $\langle p\rangle=1$. In these units length and time are dimensionless. The width of the wave packet in the momentum space is $\sigma=0.001$.

For $x>L$ these expressions take the form

$$
\begin{aligned}
\tau^{\text {Tun }}(x, t) & \\
= & \frac{M}{\langle\widehat{N}(X)\rangle} \int \mathrm{d} E\langle\mid E,+\rangle \frac{1}{2 p_{E}}|t(E)|^{2} \\
\times & {\left[2-\frac{t(E)}{t^{*}(E)} r^{*}(E) \exp \left(2 \frac{\mathrm{i}}{\hbar} p_{E} x\right)\right.} \\
& \left.-\frac{t^{*}(E)}{t(E)} r(E) \exp \left(-2 \frac{\mathrm{i}}{\hbar} p_{E} x\right)\right]\langle E,+1\rangle,
\end{aligned}
$$

$$
\begin{aligned}
\tau_{\text {corr }}^{\text {Tun }}(x, t) & \\
= & \frac{M}{2\langle\widehat{N}(X)\rangle} \int \mathrm{d} E\langle\mid E,+\rangle \frac{\mathrm{i}}{p_{E}}|t(E)|^{2} \\
& \times\left[\frac{t(E)}{t^{*}(E)} r^{*}(E) \exp \left(2 \frac{\mathrm{i}}{\hbar} p_{E} x\right)\right. \\
& \left.-\frac{t^{*}(E)}{t(E)} r(E) \exp \left(-2 \frac{\mathrm{i}}{\hbar} p_{E} x\right)\right]\langle E,+\mid\rangle .
\end{aligned}
$$

To illustrate the obtained formulae, the $\delta$-function barrier

$$
V(x)=\Omega \delta(x)
$$

and the rectangular barrier will be used. The Gaussian incident wave packet initially is far to the left of the barrier.

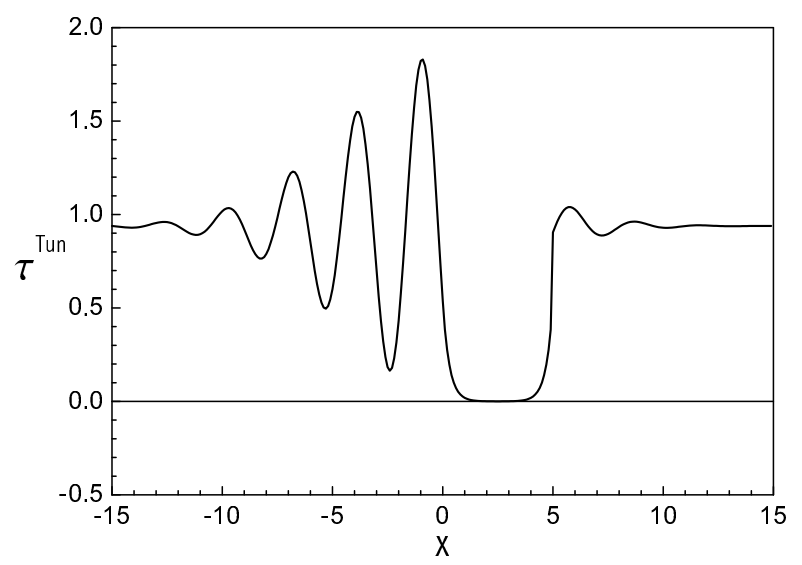

Fig. 5. Asymptotic time density for a rectangular barrier. The barrier is localized between the points $x=0$ and $x=5$ and the height of the barrier is $V_{0}=2$. The used units and parameters of the initial wave packet are the same as in Fig. 4.

In Figs. 4 and 5, we see interference-like oscillations near the barrier. Oscillations are present not only in the front of the barrier but also behind the barrier. When $x$ is far from the barrier the "time density" tends to a value close to 1 . This is in agreement with classical mechanics because in the chosen units the mean velocity of the particle is 1 . Figure 5 shows additional property of "tunnelling time density": it is almost zero in the barrier region. This explains the Hartmann and Fletcher effect [58, 59]: for opaque barriers the effective tunnelling velocity is very large.

\subsection{Reflection time}

We can easily adapt our model for the reflection, too. In doing this, one should replace the tunnelling-flag operator $\widehat{f}_{T}$ by the reflection-flag operator

$$
\widehat{f}_{R}=1-\widehat{f}_{T} .
$$

Replacement of $\widehat{f}_{T}$ by $\widehat{f}_{R}$ in Eqs. (50) and (51) gives

$$
\begin{aligned}
& \left\langle\widetilde{f}_{R}(t=\infty, X)\right\rangle \tau^{\text {Refl }}(x) \\
& \quad=\tau^{\text {Dw }}(x)-\left\langle\tilde{f}_{T}(t=\infty, X)\right\rangle \tau^{\text {Tun }}(x) .
\end{aligned}
$$

We see that in our model the important condition

$$
\tau^{\mathrm{Dw}}=T \tau^{\text {Tun }}+R \tau^{\mathrm{Refl}},
$$

where $T$ and $R$ are the transmission and reflection probabilities, is satisfied automatically. 
If the wave packet consists of waves moving in the positive direction, the density of dwell time becomes

$$
\begin{aligned}
& \tau^{\mathrm{Dw}}(x, t) \\
& \quad=2 \pi \hbar \int \mathrm{d} E\langle\mid E,+\rangle\langle E,+\mid x\rangle\langle x \mid E,+\rangle\langle E,+\mid\rangle .
\end{aligned}
$$

For $x<0$ we have

$$
\begin{aligned}
\tau^{\mathrm{Dw}}(x, t)= & M \int \mathrm{d} E\langle\mid E,+\rangle \frac{1}{p_{E}} \\
& \times\left[1+|r(E)|^{2}+r(E) \exp \left(-2 \frac{\mathrm{i}}{\hbar} p_{E} x\right)\right. \\
& \left.+r^{*}(E) \exp \left(2 \frac{\mathrm{i}}{\hbar} p_{E} x\right)\right]\langle E,+\mid\rangle, \quad \text { (65) }
\end{aligned}
$$

and for the reflection time we obtain the "time density"

$$
\begin{aligned}
\tau^{\mathrm{Refl}}(x)= & \frac{M}{1-\langle\hat{N}(X)\rangle} \int \mathrm{d} E\langle\mid E,+\rangle \frac{1}{p_{E}} \\
& \times\left[2|r(E)|^{2}+\frac{1}{2}\left(1+|r(E)|^{2}\right)\right. \\
& \times r(E) \exp \left(-2 \frac{\mathrm{i}}{\hbar} p_{E} x\right) \\
& \left.+r^{*}(E) \exp \left(2 \frac{\mathrm{i}}{\hbar} p_{E} x\right)\right]\langle E,+\mid\rangle .
\end{aligned}
$$

For $x>L$ the density of the dwell time is

$$
\tau^{\mathrm{Dw}}(x, t)=M \int \mathrm{d} E\langle\mid E,+\rangle \frac{1}{p_{E}}|t(E)|^{2}\langle E,+\mid\rangle,
$$

and the "density of the reflection time" can be expressed as

$$
\begin{aligned}
\tau^{\text {Refl }}(x)= & \frac{M}{2} \int \mathrm{d} E\langle\mid E,+\rangle \frac{1}{p_{E}}|t(E)|^{2} \\
& \times\left[\frac{t(E)}{t^{*}(E)} r^{*}(E) \exp \left(2 \frac{\mathrm{i}}{\hbar} p_{E} x\right)\right. \\
& \left.+\frac{t^{*}(E)}{t(E)} r(E) \exp \left(-2 \frac{\mathrm{i}}{\hbar} p_{E} x\right)\right]\langle E,+\mid\rangle .
\end{aligned}
$$

We will illustrate the properties of the reflection time for the same barriers and Gaussian incident wave packet initially localized far to the left from the barrier. In Figs. 6 and 7, one can see the interference-like

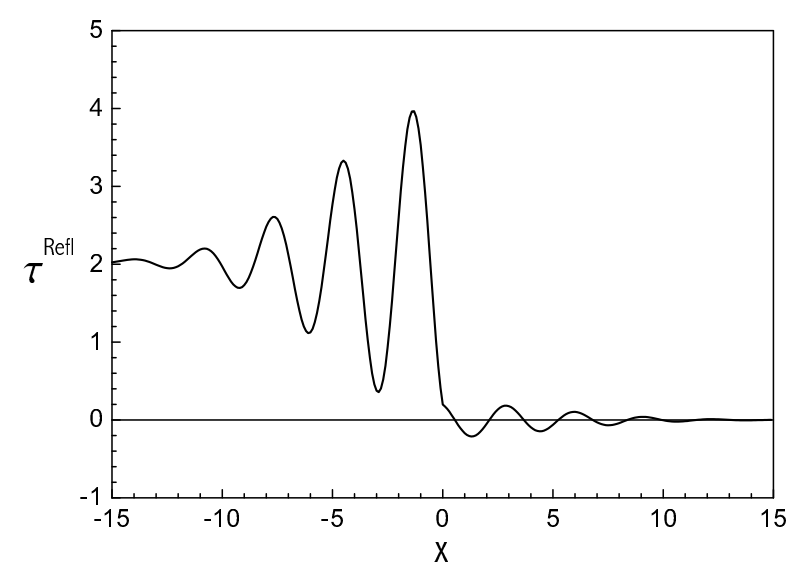

Fig. 6. Reflection time density under the same conditions as in Fig. 4.

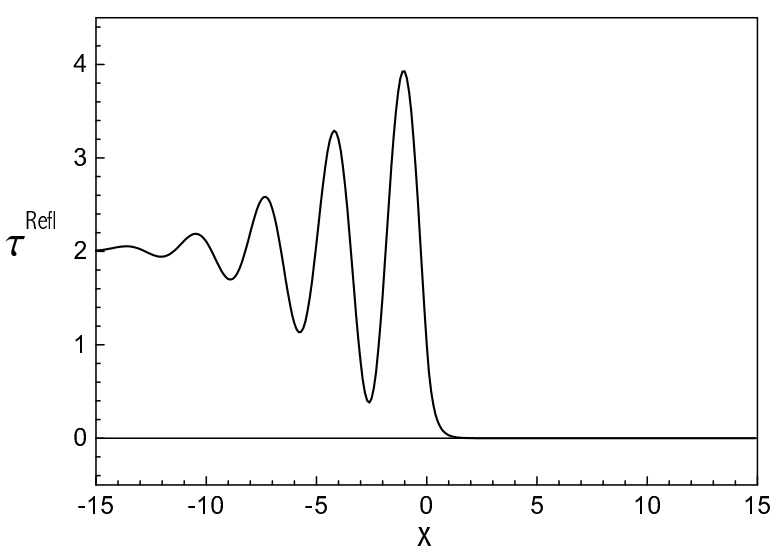

Fig. 7. Reflection time density under the same conditions as in Fig. 5.

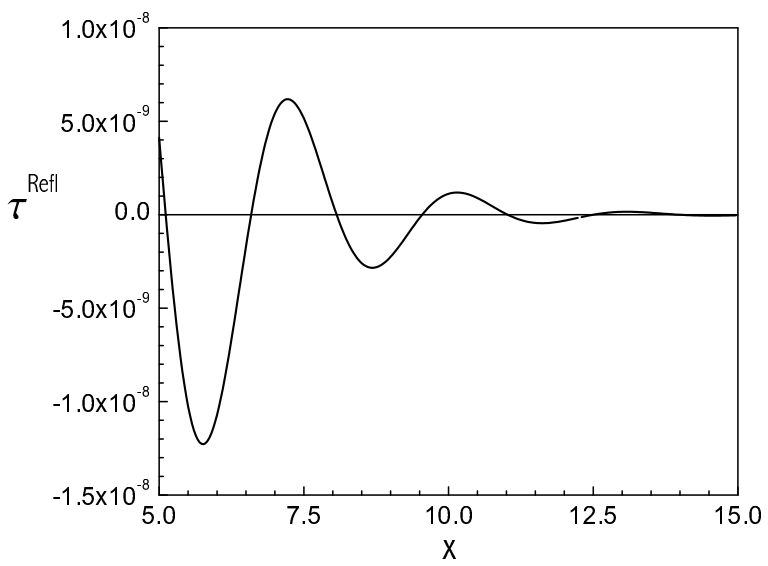

Fig. 8. Reflection time density for a rectangular barrier in the region behind the barrier. The parameters and the initial conditions are the same as in Fig. 5. 
oscillations at both sides of the barrier. Since for the rectangular barrier the "time density" behind the barrier is very small, this part is presented in Fig. 8. Behind the barrier, the "time density" at certain points becomes negative. This is because the quantity $\tau^{\operatorname{Refl}}(x)$ is not positive definite. Nonpositivity is the direct consequence of noncommutativity of the operators in Eqs. (50) and (51). There is nothing strange in the negativity of $\tau^{\text {Refl }}(x)$ because this quantity has no physical meaning. Only the integral over a large region has the meaning of time. When $x$ is far to the left from the barrier the "time density" tends to a value close to 2 and when $x$ is far to the right from the barrier the "time density" tends to 0 . This is in agreement with classical mechanics because in the chosen units, the velocity of the particle is 1 and the reflected particle crosses the area before the barrier two times.

\subsection{Asymptotic time}

As mentioned above, we can determine only the time that the tunnelling particle spends in a large region containing the barrier, i.e. the asymptotic time. In our model this time is expressed as an integral of quantity (50) over this region. We can do the integration explicitly.

The continuity equation yields

$$
\frac{\partial}{\partial t} \widetilde{D}\left(x_{\mathrm{D}}, t\right)+\frac{\partial}{\partial x_{\mathrm{D}}} \widetilde{J}\left(x_{\mathrm{D}}, t\right)=0 .
$$

The integration can be performed by parts:

$$
\begin{aligned}
& \int_{0}^{t} \widetilde{D}\left(x_{\mathrm{D}}, t_{1}\right) \mathrm{d} t_{1} \\
& \quad=t \widetilde{D}\left(x_{\mathrm{D}}, t\right)+\frac{\partial}{\partial x} \int_{0}^{t} t_{1} \widetilde{J}\left(x_{\mathrm{D}}, t_{1}\right) \mathrm{d} t_{1} .
\end{aligned}
$$

If the density matrix $\widehat{\rho}_{\mathrm{P}}(0)$ represents a localized particle then $\lim _{t \rightarrow \infty}\left(\widetilde{D}(x, t) \widehat{\rho}_{\mathrm{P}}(0)\right)=0$. Therefore, we can write an effective equality

$$
\int_{0}^{\infty} \widetilde{D}\left(x_{\mathrm{D}}, t_{1}\right) \mathrm{d} t_{1}=\frac{\partial}{\partial x} \int_{0}^{\infty} t_{1} \widetilde{J}\left(x_{\mathrm{D}}, t_{1}\right) \mathrm{d} t_{1} .
$$

We introduce the operator

$$
\widehat{T}(x)=\int_{0}^{\infty} t_{1} \widetilde{J}\left(x, t_{1}\right) \mathrm{d} t_{1} .
$$

We consider the asymptotic time, i. e. the time the particle spends between points $x_{1}$ and $x_{2}$ when $x_{1} \rightarrow-\infty$, $x_{2} \rightarrow+\infty$,

$$
t^{\text {Tun }}\left(x_{2}, x_{1}\right)=\int_{x_{1}}^{x_{2}} \tau^{\text {Tun }}(x) \mathrm{d} x .
$$

After the integration we have

$$
t^{\text {Tun }}\left(x_{2}, x_{1}\right)=t^{\text {Tun }}\left(x_{2}\right)-t^{\text {Tun }}\left(x_{1}\right),
$$

where

$$
t^{\operatorname{Tun}}(x)=\frac{1}{2\langle\widehat{N}(x)\rangle}\langle\widehat{N}(x) \widehat{T}(x)+\widehat{T}(x) \widehat{N}(x)\rangle .
$$

If we assume that the initial wave packet is far to the left from the points under the investigation and consists only of the waves moving in the positive direction, then Eq. (72) can be simplified.

In the energy representation the operator (71) is

$$
\begin{aligned}
\widehat{T}(x)= & \int_{-\infty}^{\infty} t_{1} \mathrm{~d} t_{1} \sum_{\alpha, \alpha^{\prime}} \iint \mathrm{d} E \mathrm{~d} E^{\prime}|E, \alpha\rangle \\
& \times\left\langle E, \alpha|\widehat{J}(x)| E^{\prime}, \alpha^{\prime}\right\rangle\left\langle E^{\prime}, \alpha^{\prime}\right| \\
& \times \exp \left(\frac{\mathrm{i}}{\hbar}\left(E-E^{\prime}\right) t_{1}\right) .
\end{aligned}
$$

The integration over time yields

$$
2 \mathrm{i} \pi \hbar^{2} \frac{\partial}{\partial E^{\prime}} \delta\left(E-E^{\prime}\right)
$$

and we obtain

$$
\widehat{T}(x)=-\mathrm{i} \hbar 2 \pi \hbar \sum_{\alpha, \alpha^{\prime}} \int \mathrm{d} E|E, \alpha\rangle
$$

$$
\begin{aligned}
& \times\left[\left.\frac{\partial}{\partial E^{\prime}}\left\langle E, \alpha|\widehat{J}(x)| E^{\prime}, \alpha^{\prime}\right\rangle\right|_{E^{\prime}=E}\left\langle E, \alpha^{\prime}\right|\right. \\
& \left.\quad+\left\langle E, \alpha|\widehat{J}(x)| E, \alpha^{\prime}\right\rangle \frac{\partial}{\partial E}\left\langle E, \alpha^{\prime}\right|\right]
\end{aligned}
$$

$$
\begin{aligned}
\langle\widehat{N}(X) \widehat{T}(x)\rangle \\
=-\mathrm{i} \hbar 4 \pi^{2} \hbar^{2} \\
\quad \times \sum_{\alpha} \int \mathrm{d} E\langle\Psi \mid E,+\rangle\langle E,+|\widehat{J}(X)| E, \alpha\rangle \\
\quad \times\left[\left.\frac{\partial}{\partial E^{\prime}}\left\langle E, \alpha|\widehat{J}(x)| E^{\prime},+\right\rangle\right|_{E^{\prime}=E}\right. \\
\left.\quad+\langle E, \alpha|\widehat{J}(x)| E,+\rangle \frac{\partial}{\partial E}\right]\langle E,+\mid \Psi\rangle .
\end{aligned}
$$

Substituting expressions for the matrix elements of the probability flux operator we obtain the equation 


$$
\begin{aligned}
& \langle\widehat{N}(X) \widehat{T}(x)\rangle \\
& =\int \mathrm{d} E\langle\Psi \mid E,+\rangle t^{*}(E) \frac{\hbar}{\mathrm{i}} \frac{\partial}{\partial E} t(E)\langle E,+\mid \Psi\rangle \\
& \quad+M x \int \mathrm{d} E\langle\Psi \mid E,+\rangle \frac{1}{p_{E}}|t(E)|^{2}\langle E,+\mid \Psi\rangle \\
& \quad+\mathrm{i} \hbar \frac{M}{2} \int \mathrm{d} E\langle\Psi \mid E,+\rangle \\
& \quad \times \frac{1}{p_{E}^{2}} r^{*}(E) t^{2}(E) \exp \left(2 \frac{\mathrm{i}}{\hbar} p_{E} x\right)\langle E,+\mid \Psi\rangle .
\end{aligned}
$$

When $x \rightarrow+\infty$, the last term vanishes and we have

$$
\begin{aligned}
& \langle\widehat{N}(X) \widehat{T}(x)\rangle \\
& =\int \mathrm{d} E\langle\Psi \mid E,+\rangle t^{*}(E) \frac{\hbar}{\mathrm{i}} \frac{\partial}{\partial E} t(E)\langle E,+\mid \Psi\rangle \\
& \quad+M x \int \mathrm{d} E\langle\Psi \mid E,+\rangle \frac{1}{p_{E}}|t(E)|^{2}\langle E,+\mid \Psi\rangle, \\
& \quad x \rightarrow+\infty .
\end{aligned}
$$

This expression is equal to $\langle\widehat{T}(x)\rangle$,

$$
\langle\widehat{N}(X) \widehat{T}(x)\rangle \rightarrow\langle\widehat{T}(x)\rangle, \quad x \rightarrow+\infty .
$$

When the point with coordinate $x$ is in front of the barrier, Eq. (74) becomes

$$
\begin{aligned}
&\langle\widehat{N}(X)\widehat{T}(x)\rangle \\
&=-\mathrm{i} \hbar \int \mathrm{d} E\langle\Psi \mid E,+\rangle|t(E)|^{2} \\
& \quad \times {\left[\frac{\mathrm{i}}{\hbar} \frac{M}{p_{E}} x-\frac{M}{2 p_{E}^{2}} r(E) \exp \left(-\frac{\mathrm{i}}{\hbar} 2 p_{E} x\right)+\frac{\partial}{\partial E}\right] } \\
& \quad \times\langle E,+\mid \Psi\rangle .
\end{aligned}
$$

When $|x|$ is large, the second term vanishes and we have

$$
\begin{aligned}
& \langle\widehat{N}(X) \widehat{T}(x)\rangle \\
& \rightarrow M x \int \mathrm{d} E\langle\Psi \mid E,+\rangle \frac{1}{p_{E}}|t(E)|^{2}\langle E,+\mid \Psi\rangle \\
& \quad+\int \mathrm{d} E\langle\Psi \mid E,+\rangle|t(E)|^{2} \frac{\hbar}{\mathrm{i}} \frac{\partial}{\partial E}\langle E,+\mid \Psi\rangle .
\end{aligned}
$$

The imaginary part of Eq. (76) is not zero. This means that for determination of the asymptotic time it is insufficient to integrate only in the region containing the barrier. For quasi-monochromatic wave packets from Eqs. (71)-(74) and (76) we obtain the limits

$$
\begin{aligned}
& t^{\text {Tun }}\left(x_{2}, x_{1}\right) \rightarrow t_{T}^{\mathrm{Ph}}+\frac{1}{p_{E}} M\left(x_{2}-x_{1}\right), \\
& t_{\text {corr }}^{\text {Tun }}\left(x_{2}, x_{1}\right) \rightarrow-t_{T}^{\mathrm{Im}},
\end{aligned}
$$

where

$$
t_{T}^{\mathrm{Ph}}=\hbar \frac{\mathrm{d}}{\mathrm{d} E}(\arg t(E))
$$

is the phase time and

$$
t_{T}^{\operatorname{Im}}=\hbar \frac{\mathrm{d}}{\mathrm{d} E}(\ln |t(E)|)
$$

is the imaginary part of the complex time.

In order to take the limit $x \rightarrow-\infty$ we have to perform more accurate calculations. The range of integration over time cannot be extended to $-\infty$ because such extension corresponds to the initial wave packet being infinitely far from the barrier. We can extend the range of the integration over the time to $-\infty$ only in $\widehat{N}(X)$. For $x<0$ we obtain the following equation:

$$
\begin{aligned}
& \langle\widehat{N}(X) \widehat{T}(x)\rangle \\
& =\frac{1}{4 \pi M \mathrm{i}} \int_{0}^{\infty} t \mathrm{~d} t \\
& \quad \times\left[I_{1}^{*}(x, t) \frac{\partial}{\partial x} I_{2}(x, t)-I_{2}(x, t) \frac{\partial}{\partial x} I_{1}^{*}(x, t)\right],
\end{aligned}
$$

where

$$
\begin{aligned}
I_{1}(x, t)= & \int \mathrm{d} E \frac{1}{\sqrt{p_{E}}}|t(E)|^{2} \\
& \times \exp \left[\frac{\mathrm{i}}{\hbar}\left(p_{E} x-E t\right)\right]\langle E,+\mid \Psi\rangle, \\
I_{2}(x, t)= & \int \mathrm{d} E \frac{1}{\sqrt{p_{E}}} \\
& \times\left[\exp \left(\frac{\mathrm{i}}{\hbar} p_{E} x\right)+r(E) \exp \left(-\frac{\mathrm{i}}{\hbar} p_{E} x\right)\right] \\
& \times \exp \left(-\frac{\mathrm{i}}{\hbar} E t\right)\langle E,+\mid \Psi\rangle .
\end{aligned}
$$

Here $I_{1}(x, t)$ is equal to the wave function at the point $x$ and the time moment $t$, when the propagation is in the free space and the initial wave function in the energy representation is $|t(E)|^{2}\langle E,+\mid \Psi\rangle$. When $t \geq 0$ and 


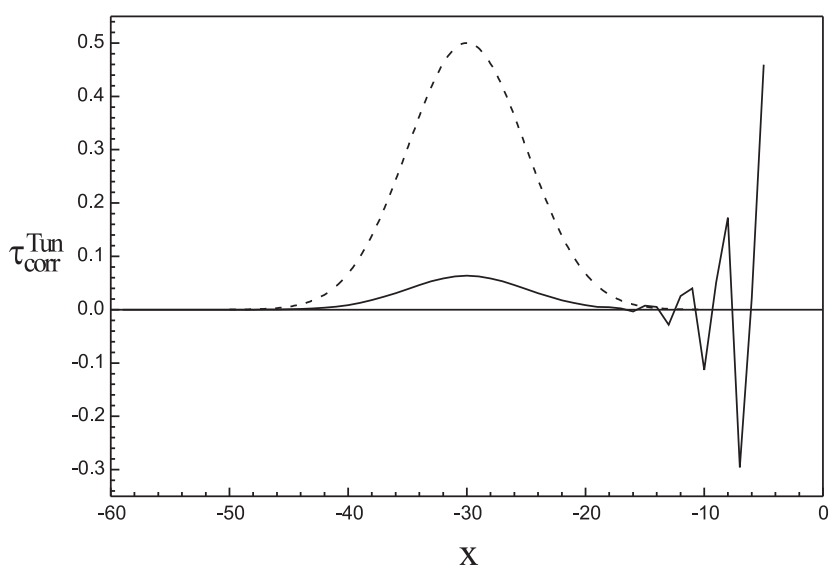

Fig. 9. The quantity $\tau_{\text {corr }}^{\text {Tun }}(x)$ for the $\delta$-function barrier with the parameters and initial conditions as in Fig. 4. The initial packet is shown by dashed line.

$x \rightarrow-\infty$, then $I_{1}(x, t) \rightarrow 0$. That is why the initial wave packet contains only the waves moving in the positive direction. Therefore, $\langle\widehat{N}(X) \widehat{T}(x)\rangle \rightarrow 0$ when $x \rightarrow-\infty$. From this analysis it follows that the region in which the asymptotic time is well determined has to include not only the barrier but also the initial wave packet region.

In such a case from Eqs. (72) and (73) we obtain expression for the asymptotic time

$$
\begin{aligned}
& t^{\text {Tun }}\left(x_{2}, x_{1} \rightarrow-\infty\right) \\
& =\frac{1}{\langle\widehat{N}(X)\rangle} \int \mathrm{d} E\langle\Psi \mid E,+\rangle t^{*}(E) \\
& \quad \times\left(\frac{M}{p_{E}} x_{2}-\mathrm{i} \hbar \frac{\partial}{\partial E}\right) t(E)\langle E,+\mid \Psi\rangle .
\end{aligned}
$$

From Eq. (75) it follows that

$$
t^{\operatorname{Tun}}\left(x_{2}, x_{1} \rightarrow-\infty\right)=\frac{1}{\langle\widehat{N}(X)\rangle}\left\langle\widehat{T}\left(x_{2}\right)\right\rangle
$$

where $\widehat{T}\left(x_{2}\right)$ is defined as the probability flux integral (71). Equations (84) and (85) give the same value for tunnelling time as does an approach in [60,61].

The integral of the quantity $\tau_{\text {corr }}^{\text {Tun }}(x)$ over a large region is zero. We have seen that it is not enough to choose the region around the barrier - this region has to include also the initial wave packet location. This fact will be illustrated by numerical calculations.

The quantity $\tau_{\text {corr }}^{\text {Tun }}(x)$ for $\delta$-function barrier is represented in Fig. 9. We see that $\tau_{\text {corr }}^{\text {Tun }}(x)$ is not equal to zero not only in the region around the barrier but also it is not zero in the location of the initial wave packet. For

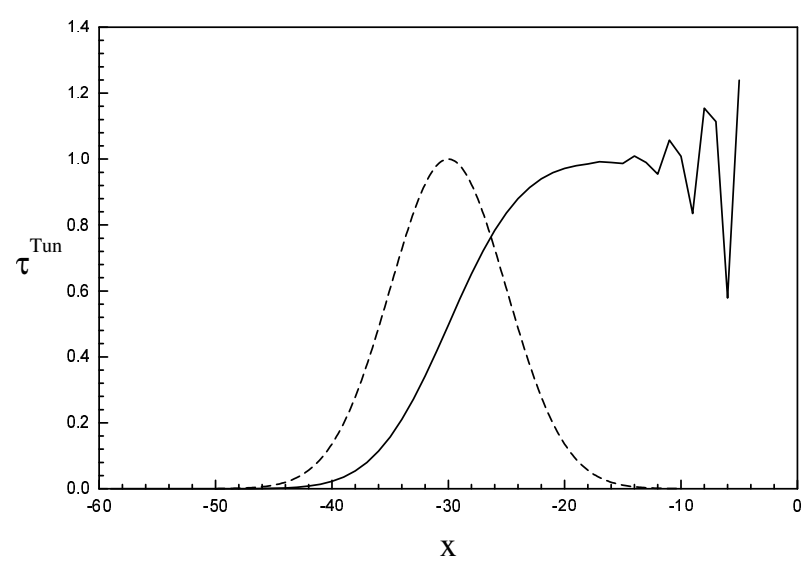

Fig. 10. Tunnelling time density for the same conditions and parameters as in Fig. 9.

comparison, the quantity $\tau^{\text {Tun }}(x)$ for the same conditions is presented in Fig. 10.

\section{Arrival time}

The detection of the particles in time-of-flight and coincidence experiments are common, and quantum mechanics should give a method for the calculation of the arrival time. The arrival time distribution may be useful in solving the tunnelling time problem as well. Therefore, the quantum description of arrival time has attracted much attention [62-77].

Aharonov and Bohm introduced the arrival time operator [62]

$$
\widehat{T}_{\mathrm{AB}}=\frac{m}{2}\left[(X-\widehat{x}) \frac{1}{\widehat{p}}+\frac{1}{\widehat{p}}(X-\widehat{x})\right] .
$$

By imposing several conditions (normalization, positivity, minimum variance, and symmetry with respect to the arrival point $X$ ) a quantum arrival time distribution for a free particle was obtained by Kijowski [63]. Kijowski's distribution may be associated with the positive valued operator measure generated by the eigenstates of $\widehat{T}_{\mathrm{AB}}$. However, Kijowski's set of conditions cannot be applied in a general case [63]. Nevertheless, arrival time operators can be constructed even if the particle is not free $[73,78]$.

Since the mean arrival time even in classical mechanics can be infinite or the particle may not arrive at all, it is convenient to deal not with the mean arrival time and the corresponding operator $\widehat{T}$, but with the probability distribution of the arrival times [24]. The probability distribution of the arrival times can be obtained from a suitable classical definition. The noncommutativity of the operators in quantum mechanics 
is circumvented by using the concept of weak measurements.

\subsection{Arrival time in classical mechanics}

In classical mechanics the particle moves along the trajectory $H(x, p)=$ const as $t$ increases. This allows us to work out the time of arrival at the point $x(t)=X$, by identifying the point $\left(x_{0}, p_{0}\right)$ of the phase space where the particle is at $t=0$, and then following the trajectory that passes by this point, up to arrival at the point $X$. If multiple crossings are possible, one may define a distribution of arrival times with contributions from all crossings, when no distinction is made between first, second, and $n$th arrivals. In this article we will consider such a distribution.

We can ask whether there is a definition of the arrival time that is valid in both classical and quantum mechanics. In our opinion, the words "the particle arrives from the left at the point $X$ at the time $t$ " mean that:

(i) at time $t$ the particle was in the region $x<X$ and

(ii) at time $t+\Delta t(\Delta t \rightarrow 0)$ the particle is found in the region $x>X$.

Now we apply the definition given by (i) and (ii) to the time of arrival in the classical case.

Since quantum mechanics deals with probabilities, it is convenient to use probabilistic description of the classical mechanics, as well. Therefore, we will consider an ensemble of noninteracting classical particles. The probability density in the phase space is $\rho(x, p ; t)$.

Let us denote the region $x<X$ as $\Gamma_{1}$ and the region $x>X$ as $\Gamma_{2}$. The probability that the particle arrives from region $\Gamma_{1}$ to region $\Gamma_{2}$ at a time between $t$ and $t+\Delta t$ is proportional to the probability that the particle is in region $\Gamma_{1}$ at time $t$ and in region $\Gamma_{2}$ at time $t+\Delta t$. This probability is

$$
\Pi_{+}(t) \Delta t=\frac{1}{N_{+}} \int_{\Omega} \mathrm{d} p \mathrm{~d} x \rho(x, p ; t),
$$

where $N_{+}$is the constant of normalization and the region of phase space $\Omega$ has the following properties: (i) the coordinates of the points in $\Omega$ are in the space region $\Gamma_{1}$ and (ii) if the phase trajectory goes through a point of the region $\Omega$ at time $t$ then the particle at time $t+\Delta t$ is in the space region $\Gamma_{2}$. Since $\Delta t$ is infinitesimal, the change of coordinate during the time interval $\Delta t$ is equal to $(p / m) \Delta t$. Therefore, the particle arrives from region $\Gamma_{1}$ to region $\Gamma_{2}$ only if the momentum of the particle at the point $X$ is positive. The phase space region $\Omega$ consists of the points with positive momentum $p$ and with coordinates between $X-(p / m) \Delta t$ and $X$. Then from Eq. (87) we have the probability of arrival time

$$
\Pi_{+}(t) \Delta t=\frac{1}{N_{+}} \int_{0}^{\infty} \mathrm{d} p \int_{X-(p / m) \Delta t}^{X} \mathrm{~d} x \rho(x, p ; t) .
$$

Since $\Delta t$ is infinitesimal and the momentum of every particle is finite, we can replace $x$ in Eq. (88) by $X$ and obtain the equality

$$
\Pi_{+}(t, X)=\frac{1}{N_{+}} \int_{0}^{\infty} \frac{p}{m} \rho(X, p ; t) \mathrm{d} p .
$$

The obtained arrival time distribution $\Pi_{+}(t, X)$ is well known and has appeared quite often in the literature (see, e. g., the review [73] and references therein).

The probability current in classical mechanics is

$$
J(x ; t)=\int_{-\infty}^{+\infty} \frac{p}{m} \rho(x, p ; t) \mathrm{d} p .
$$

From Eqs. (89) and (90) it is clear that the time of arrival is related to the probability current. This relation, however, is not straightforward. We can introduce the "positive probability current"

$$
J_{+}(x ; t)=\int_{0}^{\infty} \frac{p}{m} \rho(x, p ; t) \mathrm{d} p
$$

and rewrite Eq. (89) as

$$
\Pi_{+}(t, X)=\frac{1}{N_{+}} J_{+}(X ; t) .
$$

The proposed [79-81] various quantum versions of $J_{+}$ even in the case of the free particle can be negative (the so-called backflow effect). Therefore, the classical expression (92) for the time of arrival becomes problematic in quantum mechanics.

Similarly, for arrival from the right we obtain the probability density

$$
\Pi_{-}(t, X)=\frac{1}{N_{-}} J_{-}(X ; t),
$$

where the negative probability current is

$$
J_{-}(x ; t)=\int_{-\infty}^{0} \frac{|p|}{m} \rho(x, p ; t) \mathrm{d} p .
$$

We see that our definition given at the beginning of this section leads to the proper result in classical mechanics. The conditions (i) and (ii) do not involve the concept of the trajectories. We can try to use this definition also in quantum mechanics. 


\subsection{Weak measurement of the arrival time}

The proposed definition of the arrival time probability distribution can be used in quantum mechanics only if the determination of the region in which the particle is does not disturb the motion of the particle. This can be achieved using the weak measurements of Aharonov, Albert, and Vaidman [16-21].

We use the weak measurement, described in Section 2. The detector interacts with the particle only in region $\Gamma_{1}$. As regards the operator $\widehat{A}$ we take the projection operator $\widehat{P}_{1}$ which projects into region $\Gamma_{1}$. In analogy to [17], we define the "weak value" of the probability of finding the particle in the region $\Gamma_{1}$,

$$
W(1) \equiv\left\langle\widehat{P}_{1}\right\rangle=\frac{\left\langle\widehat{p}_{q}\right\rangle_{0}-\left\langle\widehat{p}_{q}\right\rangle}{\lambda \tau} .
$$

In order to obtain the arrival time probability using the definition from Section 5.1, we measure the momenta $p_{q}$ of each detector after the interaction with the particle. After time $\Delta t$ we perform the final, postselection measurement on the particles of our ensemble and measure if the particle is found in region $\Gamma_{2}$. Then we collect the outcomes $p_{q}$ only for the particles found in region $\Gamma_{2}$.

The projection operator projecting into the region $\Gamma_{2}$ is $\widehat{P}_{2}$. In the Heisenberg representation this operator is

$$
\widetilde{P}_{2}(t)=\widehat{U}(t)^{\dagger} \widehat{P}_{2} \widehat{U}(t)
$$

where $\widehat{U}$ is the evolution operator of the free particle. Taking the operator $B$ from Section 2 as $\widetilde{P}_{2}(\Delta t)$ and using Eq. (95) we can introduce a weak value $W(1 \mid 2)$ of probability to find the particle in the region $\Gamma_{1}$ on condition that the particle after time $\Delta t$ is in the region $\Gamma_{2}$. The probability that the particle is in region $\Gamma_{1}$ and after time $\Delta t$ it is in region $\Gamma_{2}$ then equals

$$
W(1,2)=W(2) W(1 \mid 2) \text {. }
$$

When the measurement time $\tau$ is sufficiently small, the influence of the Hamiltonian of the particle can be neglected. Using Eq. (7) from Section 2 we obtain

$$
\begin{aligned}
W(1,2) \approx & \frac{1}{2}\left\langle\widetilde{P}_{2}(\Delta t) \widehat{P}_{1}+\widehat{P}_{1} \widetilde{P}_{2}(\Delta t)\right\rangle \\
& +\frac{\mathrm{i}}{\hbar}\left(\left\langle\widehat{p}_{q}\right\rangle\langle\widehat{q}\rangle-\operatorname{Re}\left\langle\widehat{q}_{q}\right\rangle\right)\left\langle\left[\widehat{P}_{1}, \widetilde{P}_{2}(\Delta t)\right]\right\rangle .
\end{aligned}
$$

The probability $W(1,2)$ is constructed using conditions (i) and (ii) from Section 5.1: the weak measurement is performed to determine if the particle is in the region $\Gamma_{1}$ and after time $\Delta t$ the strong measurement determines if the particle is in the region $\Gamma_{2}$. Therefore, according to Section 5.1 , the quantity $W(1,2)$ after normalization can be considered as the weak value of the arrival time probability distribution.

Equation (98) consists of two terms and we accordingly can introduce two quantities

$$
\begin{aligned}
& \Pi^{(1)}=\frac{1}{2 \Delta t}\left\langle\widehat{P}_{1} \widetilde{P}_{2}(\Delta t)+\widetilde{P}_{2}(\Delta t) \widehat{P}_{1}\right\rangle, \\
& \Pi^{(2)}=\frac{1}{2 \mathrm{i} \Delta t}\left\langle\left[\widehat{P}_{1}, \widetilde{P}_{2}(\Delta t)\right]\right\rangle .
\end{aligned}
$$

Then

$$
W(1,2)=\Pi^{(1)} \Delta t-\frac{2 \Delta t}{\hbar}\left(\left\langle\widehat{p}_{q}\right\rangle\langle\widehat{q}\rangle-\operatorname{Re}\left\langle\widehat{q}_{q}\right\rangle\right) \Pi^{(2)} .
$$

If the commutator $\left[\widehat{P}_{1}, \widetilde{P}_{2}(\Delta t)\right]$ in Eqs. (99)-(101) is not zero, then, even in the limit of the very weak measurement, the measured value depends on the particular detector. This fact means that in such a case we cannot obtain a definite value for the arrival time probability. Moreover, the coefficient $\left(\left\langle\widehat{p}_{q}\right\rangle\langle\widehat{q}\rangle-\operatorname{Re}\left\langle\widehat{q}_{q}\right\rangle\right)$ may be zero for a specific initial state of the detector, e. g., for a Gaussian distribution of the coordinate $q$ and momen$\operatorname{tum} p_{q}$.

The quantities $W(1,2), \Pi^{(1)}$, and $\Pi^{(2)}$ are real. However, it is convenient to consider the complex quantity

$$
\Pi_{C}=\Pi^{(1)}+\mathrm{i} \Pi^{(2)}=\frac{1}{\Delta t}\left\langle\widehat{P}_{1} \widetilde{P}_{2}(\Delta t)\right\rangle .
$$

We call it the "complex arrival probability". We can introduce the corresponding operator

$$
\widehat{\Pi}_{+}=\frac{1}{\Delta t} \widehat{P}_{1} \widetilde{P}_{2}(\Delta t) \text {. }
$$

By analogy, the operator

$$
\widehat{\Pi}_{-}=\frac{1}{\Delta t} \widehat{P}_{2} \widetilde{P}_{1}(\Delta t)
$$

corresponds to arrival from the right.

The introduced operator $\widehat{\Pi}_{+}$has some of the properties of the classical positive probability current. From the conditions $\widehat{P}_{1}+\widehat{P}_{2}=1$ and $\widetilde{P}_{1}(\Delta t)+\widetilde{P}_{2}(\Delta t)=1$ we have

$$
\widehat{\Pi}_{+}-\widehat{\Pi}_{-}=\frac{1}{\Delta t}\left(\widetilde{P}_{2}(\Delta t)-\widehat{P}_{2}\right)
$$

In the limit $\Delta t \rightarrow 0$ we obtain the probability current $\widehat{J}=\lim _{\Delta t \rightarrow 0}\left(\widehat{\Pi}_{+}-\widehat{\Pi}_{-}\right)$, as in classical mechanics. However, the quantity $\left\langle\widehat{\Pi}_{+}\right\rangle$is complex and the real part can be negative, in contrast to the classical quantity $J_{+}$. The reason for this is the noncommutativity of the operators $\widehat{P}_{1}$ and $\widetilde{P}_{2}(\Delta t)$. When the imaginary part 
is small, the quantity $\left\langle\widehat{\Pi}_{+}\right\rangle$after normalization can be considered as the approximate probability distribution of the arrival time.

\subsection{Arrival time probability}

The operator $\widehat{\Pi}_{+}$was obtained without specification of the Hamiltonian of the particle and is suitable for free particles and for particles subjected to an external potential as well. In this section we consider the arrival time of the free particle.

The calculation of the "weak arrival time distribution" $W(1,2)$ involves the average value $\left\langle\widehat{\Pi}_{+}\right\rangle$. Therefore, it is useful to have the matrix elements of the operator $\widehat{\Pi}_{+}$. It should be noted that the matrix elements of the operator $\widehat{\Pi}_{+}$, as well as the operator itself, are only auxiliary quantities and do not have an independent meaning.

In the basis of the momentum eigenstates $|p\rangle$, normalized according to the condition $\left\langle p_{1} \mid p_{2}\right\rangle=$ $2 \pi \hbar \delta\left(p_{1}-p_{2}\right)$, the matrix elements of the operator $\widehat{\Pi}_{+}$ are

$$
\begin{aligned}
\left\langle p_{1}\left|\widehat{\Pi}_{+}\right| p_{2}\right\rangle & \\
= & \frac{1}{\Delta t}\left\langle p_{1}\left|\widehat{P}_{1} \widehat{U}(\Delta t)^{\dagger} \widehat{P}_{2} \widehat{U}(\Delta t)\right| p_{2}\right\rangle \\
= & \frac{1}{\Delta t} \int_{-\infty}^{X} \mathrm{~d} x_{1} \int_{X}^{\infty} \mathrm{d} x_{2} \exp \left(-\frac{\mathrm{i}}{\hbar} p_{1} x_{1}\right) \\
& \times\left\langle x_{1}\left|\widehat{U}(\Delta t)^{\dagger}\right| x_{2}\right\rangle \exp \left(\frac{\mathrm{i}}{\hbar} p_{2} x_{2}-\frac{\mathrm{i}}{\hbar} \frac{p_{2}^{2}}{2 m} \Delta t\right) .
\end{aligned}
$$

After performing the integration one obtains

$$
\begin{aligned}
& \left\langle p_{1}\left|\widehat{\Pi}_{+}\right| p_{2}\right\rangle \\
& =\frac{\mathrm{i} \hbar}{2 \Delta t\left(p_{2}-p_{1}\right)} \exp \left[\frac{\mathrm{i}}{\hbar}\left(p_{2}-p_{1}\right) X\right] \\
& \quad \times\left[\exp \left(\frac{\mathrm{i}}{\hbar} \frac{\Delta t}{2 m}\left(p_{1}^{2}-p_{2}^{2}\right)\right) \operatorname{erfc}\left(-p_{1} \sqrt{\frac{\mathrm{i} \Delta t}{2 \hbar m}}\right)\right. \\
& \left.\quad-\operatorname{erfc}\left(-p_{2} \sqrt{\frac{\mathrm{i} \Delta t}{2 \hbar m}}\right)\right],
\end{aligned}
$$

where $\sqrt{\mathrm{i}}=\exp (\mathrm{i} \pi / 4)$. When

$$
\frac{1}{\hbar} \frac{\Delta t}{2 m}\left(p_{1}^{2}-p_{2}^{2}\right) \ll 1
$$

$$
\begin{gathered}
p_{1} \sqrt{\frac{\Delta t}{2 \hbar m}}>1, \\
p_{2} \sqrt{\frac{\Delta t}{2 \hbar m}}>1,
\end{gathered}
$$

the matrix elements of the operator $\widehat{\Pi}_{+}$are

$$
\left\langle p_{1}\left|\widehat{\Pi}_{+}\right| p_{2}\right\rangle \approx \frac{p_{1}+p_{2}}{2 m} \exp \left[\frac{\mathrm{i}}{\hbar}\left(p_{2}-p_{1}\right) X\right] \text {. }
$$

This equation coincides with the expression for the matrix elements of the probability current operator.

From Eq. (106) we obtain the diagonal matrix elements of the operator $\widehat{\Pi}_{+}$,

$$
\begin{aligned}
\left\langle p\left|\widehat{\Pi}_{+}\right| p\right\rangle= & \frac{p}{2 m} \operatorname{erfc}\left(-p \sqrt{\frac{\mathrm{i} \Delta t}{2 \hbar m}}\right) \\
& +\frac{\hbar}{\sqrt{\mathrm{i} 2 \pi \hbar m \Delta t}} \exp \left(-\frac{\mathrm{i}}{\hbar} \frac{p^{2}}{2 m} \Delta t\right) .
\end{aligned}
$$

The real part of the quantity $\left\langle p\left|\widehat{\Pi}_{+}\right| p\right\rangle$ is shown in Fig. 11 and the imaginary part in Fig. 12.

Using the asymptotic expressions for the error function erfc we obtain from Eq. (108) that

$$
\lim _{p \rightarrow+\infty}\left\langle p\left|\widehat{\Pi}_{+}\right| p\right\rangle \rightarrow \frac{p}{m}
$$

and $\left\langle p\left|\widehat{\Pi}_{+}\right| p\right\rangle \rightarrow 0$, when $p \rightarrow-\infty$, i.e. the imaginary part tends to zero and the real part approaches the corresponding classical value as the modulus of the momentum $|p|$ increases. Such behaviour is evident from Figs. 11 and 12 also.

The asymptotic expressions for the function erfc are valid when its argument is large, i. e.

$$
|p| \sqrt{\frac{\Delta t}{(2 \hbar m)}}>1 \quad \text { or } \quad \Delta t>\frac{\hbar}{E_{k}} .
$$

Here $E_{k}$ is the kinetic energy of the particle.

The dependence of the quantity $\operatorname{Re}\left\langle p\left|\widehat{\Pi}_{+}\right| p\right\rangle$ on $\Delta t$ is shown in Fig. 13. For small $\Delta t$ the quantity $\left\langle p\left|\widehat{\Pi}_{+}\right| p\right\rangle$ is proportional to $1 / \sqrt{\Delta t}$. Therefore, unlike in classical mechanics, in quantum mechanics $\Delta t$ cannot be zero. Equation (109) imposes the lower bound on the resolution time $\Delta t$. It follows that our model does not permit determination of the arrival time with resolution greater than $\hbar / E_{k}$. A relation similar to Eq. (109) based on measurement models was obtained by Aharonov et al. [30]. The time-energy uncertainty relations associated with the time of arrival distribution are also discussed in $[69,82]$. 


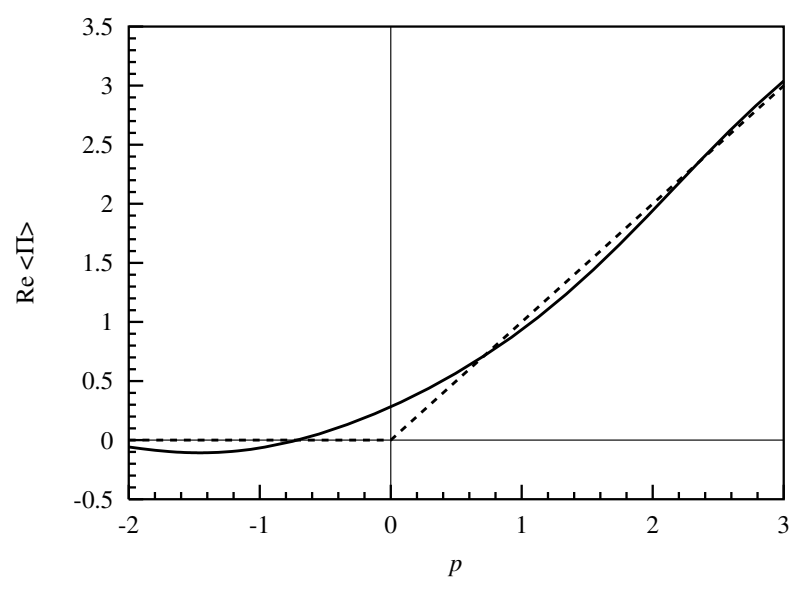

Fig. 11. The real part of the quantity $\left\langle p\left|\widehat{\Pi}_{+}\right| p\right\rangle$, according to Eq. (108). The corresponding classical positive probability current is shown with the dashed line. The parameters used are $\hbar=1$, $m=1$, and $\Delta t=1$. In this system of units, the momentum $p$ is dimensionless.

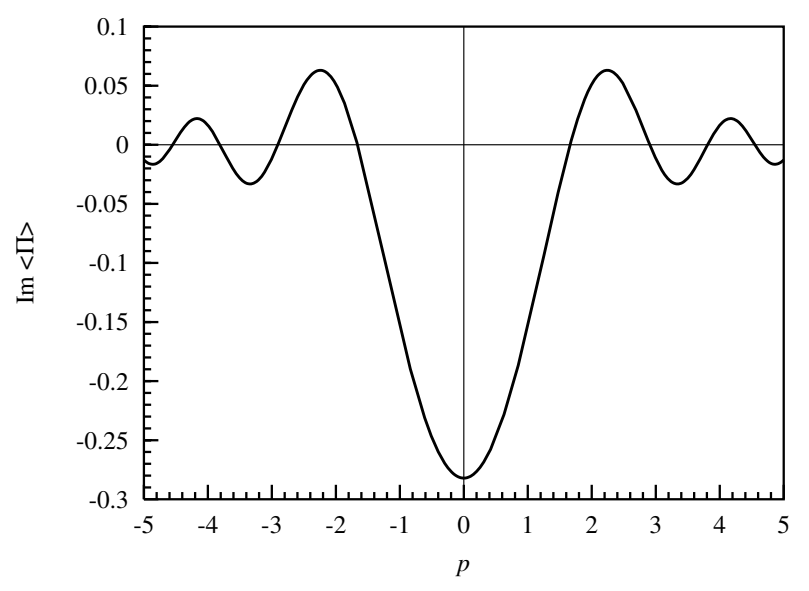

Fig. 12. The imaginary part of the quantity $\left\langle p\left|\widehat{\Pi}_{+}\right| p\right\rangle$. The parameters used are the same as in Fig. 11.

\section{Summary}

The review and generalization of the theoretical analysis of the time problem in quantum mechanics and weak measurements are presented. The tunnelling time problem is part of this more general problem. The problem of time is solved adapting the weak measurement theory to the measurement of time. In this model the expression (13) for the duration, when the arbitrary observable $\chi$ has a certain value, is obtained. This result is in agreement with the known results for the dwell time in the tunnelling time problem.

Further we consider the problem of the duration when the observable $\chi$ has a certain value on condition that the system is in a given final state. Our model of measurement allows us to obtain the expression (15) of

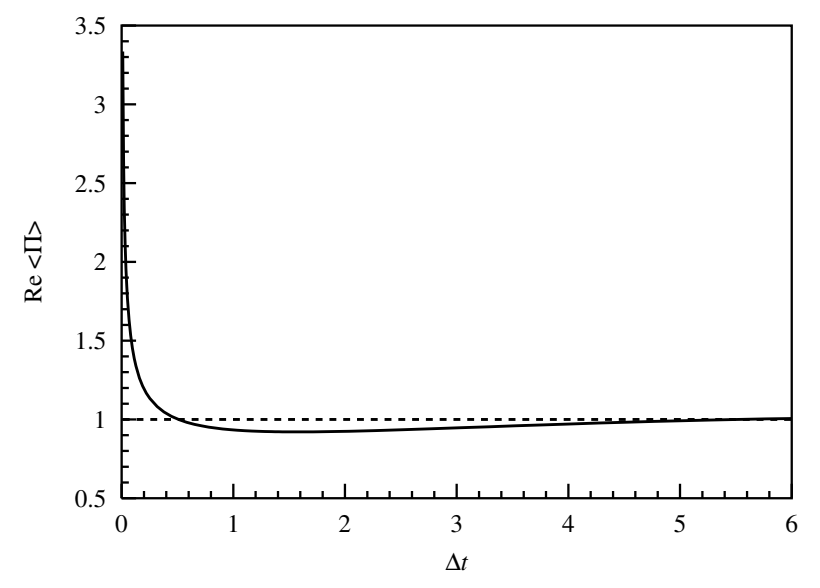

Fig. 13. The dependence of the quantity $\operatorname{Re}\left\langle p\left|\widehat{\Pi}_{+}\right| p\right\rangle$ according to Eq. (108) on the resolution time $\Delta t$. The corresponding classical positive probability current is shown with the dashed line. The parameters used are $\hbar=1, m=1$, and $p=1$. In these units, the time $\Delta t$ is dimensionless.

this duration as well. This expression has many properties of the corresponding classical time. However, such a duration not always has reasonable meaning. It is possible to obtain the duration that the quantity $\chi$ has a certain value on condition that the system is in a given final state only when the condition (19) is fulfilled. In the opposite case, there is a dependence in the outcome of the measurements on particular detector even in an ideal case, and therefore, it is impossible to obtain the definite value of the duration. When the condition (19) is not fulfilled, we introduce two quantities (16) and (17), characterizing the conditional time. These quantities are useful in the case of tunnelling and we suppose that they can be useful also for other problems.

In order to investigate the tunnelling time problem, we consider a procedure of time measurement, proposed by Steinberg [35]. This procedure shows clearly the consequences of noncommutativity of the operators and the possibility of determination of the asymptotic time. Our model also reveals the Hartmann and Fletcher effect, i. e. for opaque barriers the effective velocity is very large because the contribution of the barrier region to the time is almost zero. We cannot determine whether this velocity can be larger than $c$ because for this purpose one has to use a relativistic equation (e. g., the Dirac equation).

The definition of density of one-sided arrivals is proposed. This definition is extended to quantum mechanics, using the concept of weak measurements by Aharonov et al. [16-21]. The proposed procedure is suitable for free particles and for the particles sub- 
jected to an external potential as well. It gives not only a mathematical expression for the arrival time probability distribution but also a way of measuring the quantity obtained. However, this procedure gives no unique expression for the arrival time probability distribution.

In analogy with the complex tunnelling time, the complex arrival time "probability distribution" is introduced (Eq. (102)). It is shown that the proposed approach imposes an inherent limitation, Eq. (109), on the resolution time of the arrival time determination.

\section{References}

[1] L.A. MacColl, Phys. Rev. 40, 621 (1932).

[2] E.H. Hauge and J.A. Støvneng, Rev. Mod. Phys. 61, 917 (1989).

[3] V.S. Olkhovsky and E. Recami, Phys. Rep. 214, 339 (1992).

[4] R. Landauer and T. Martin, Rev. Mod. Phys. 66, 217 (1994).

[5] R.Y. Chiao and M. Steinberg, in: Progress in Optics, Vol. 37, ed. E. Wolf (Elsevier, Amstedram, 1997) p. 345.

[6] P. Guéret, E. Marclay, and H. Meier, Appl. Phys. Lett. 53, 1617 (1988).

[7] P. Guéret, E. Marclay, and H. Meier, Solid State Commun. 68, 977 (1988).

[8] D. Esteve et al., Physica Scripta T 29, 121 (1989).

[9] A. Enders and G. Nimtz, J. Phys. (France) 1(3), 1089 (1993).

[10] A. Ranfagni, P. Fabeni, G.P. Pazzi, and D. Mugnai, Phys. Rev. E 48, 1453 (1993).

[11] C. Spielmann, R. Szipöcs, A. Stingl, and F. Krausz, Phys. Rev. Lett. 73, 2308 (1994).

[12] W. Heitmann and G. Nimtz, Phys. Lett. A 196, 154 (1994).

[13] P. Balcou and L. Dutriaux, Phys. Rev. Lett. 78, 851 (1997).

[14] J.C. Garrison, M.W. Mitchell, R.Y. Chiao, and E.L. Bolda, Phys. Lett. A 254, 19 (1998).

[15] J.C. Martinez and E. Polatdemir, Appl. Phys. Lett. 84, 1320 (2004).

[16] Y. Aharonov, D. Albert, A. Casher, and L. Vaidman, Phys. Lett. A 124, 199 (1987).

[17] Y. Aharonov and L.V.D.Z. Albert, Phys. Rev. Lett. 60, 1351 (1988).

[18] I.M. Duck, P.M. Stevenson, and E.C.G. Sudarshan, Phys. Rev. D 40, 2112 (1989).

[19] Y. Aharonov and L. Vaidman, Phys. Rev. A 41, 11 (1990).

[20] Y. Aharonov and L. Vaidman, J. Phys. A 24, 2315 (1991).

[21] Y. Aharonov and L. Vaidman, Physica Scripta T 76, 85 (1998).
[22] J. Ruseckas and B. Kaulakys, Phys. Lett. A 287, 297 (2001).

[23] J. Ruseckas, Phys. Rev. A 63, 052107 (2001).

[24] J. Ruseckas and B. Kaulakys, Phys. Rev. A 66, 052106 (2002).

[25] J. Ruseckas and B. Kaulakys, Phys. Rev. A 63, 062103 (2001).

[26] J. Ruseckas and B. Kaulakys, Phys. Rev. A 69, 032104 (2004).

[27] J. Ruseckas, Phys. Lett. A 291, 185 (2001).

[28] J. Ruseckas, Phys. Rev. A 66, 012105 (2002).

[29] J. von Neumann, Mathematische Grundlagen der Quantenmechanik (Springer, Berlin, 1932).

[30] Y. Aharonov et al., Phys. Rev. A 57, 4130 (1998).

[31] E. Joos, Phys. Rev. D 29, 1626 (1984).

[32] C.M. Caves and G.J. Milburn, Phys. Rev. A 36, 5543 (1987).

[33] G.J. Milburn, J. Opt. Soc. Am. B 5, 1317 (1988).

[34] M.J. Gagen, H.M. Wiseman, and G.J. Milburn, Phys. Rev. A 48, 132 (1993).

[35] A.M. Steinberg, Phys. Rev. A 52, 32 (1995).

[36] M. Goto et al., J. Phys. A 37, 3599 (2004).

[37] H.G. Winful, Phys. Rev. Lett. 91, 260401 (2003).

[38] D. Sokolovski and L.M. Baskin, Phys. Rev. A 36, 4604 (1987).

[39] M. Büttiker and R. Landauer, Phys. Rev. Lett. 49, 1739 (1982).

[40] C.R. Leavens, Solid State Commun. 74, 923 (1990).

[41] C.R. Leavens, Solid State Commun. 76, 253 (1990).

[42] C.R. Leavens and G. Aers, in: Scanning Tunneling Microscopy, Vol. 3, eds. R. Weisendanger and H.-J. Güntherodt (Springer, Berlin, 1993) p. 105.

[43] C.R. Leavens, Phys. Lett. A 178, 27 (1993).

[44] C. R. Leavens, Found. Phys. 25, 229 (1995).

[45] Y. Aharonov, N. Erez, and M.O. Scully, Physica Scripta 69, 81 (2004).

[46] J.G. Muga, S. Brouard, and R. Sala, Phys. Lett. A 167, 24 (1992).

[47] A. I. Baz', Sov. J. Nucl. Phys. 4, 182 (1967).

[48] A. Ranfagni, R. Ruggeri, and A. Agresti, Found. Phys. 28, 515 (1998).

[49] A. Ranfagni, R. Ruggeri, C. Susini, A. Agresti, and P. Sandri, Phys. Rev. E 63, 025102 (2001).

[50] A. Ranfagni, R. Ruggeri, D. Mugnai, A. Agresti, C. Ranfagni, and P. Sandri, Phys. Rev. E 67, 066611 (2003).

[51] K. Hara and I. Ohba, Phys. Rev. A 67, 052105 (2003).

[52] P. Harsko, Found. Phys. 33, 1009 (2003).

[53] R.S. Dumont and T.L. Marchioro II, Phys. Rev. A 47, 85 (1993).

[54] D. Sokolovski and J.N.L. Connor, Phys. Rev. A 47, 4677 (1993).

[55] N. Yamada, Phys. Rev. Lett. 83, 3350 (1999).

[56] S. Brouard, R. Sala, and J.G. Muga, Europhys. Lett. 22, 159 (1993). 
[57] G. Iannaccone, in: Proceedings of Adriatico Research Conference on Tunneling and Its Implications, eds. D. Mugnai, A. Ranfagni, and L.S. Schulman (World Scientific, Singapore, 1997) pp. 292-309.

[58] T.E. Hartmann, J. Appl. Phys. 33, 3427 (1962).

[59] J.R. Fletcher, J. Phys. C 18, L55 (1985).

[60] V. Delgado and J.G. Muga, Phys. Rev. A 56, 3425 (1997).

[61] N. Grot, C. Rovelli, and R.S. Tate, Phys. Rev. A 54, 4676 (1996).

[62] Y. Aharonov and D. Bohm, Phys. Rev. 122, 1649 (1961).

[63] J. Kijowski, Rep. Math. Phys. 6, 361 (1974).

[64] J.G. Muga, C.R. Leavens, and J.P. Palao, Phys. Rev. A 58, 4336 (1998).

[65] A.D. Baute, I.L. Egusquiza, and J.G. Muga, Phys. Rev. A 64, 012501 (2001).

[66] K.-I. Aoki, A. Horikoshi, and E. Nakamura, Phys. Rev. A 62, 022101 (2000).

[67] A.D. Baute, I.L. Egusquiza, and J.G. Muga, Phys. Rev. A 64, 014101 (2001).

[68] A.D. Baute, I.L. Egusquiza, J.G. Muga, and R. SalaMayato, Phys. Rev. A 61, 052111 (2000).

[69] A.D. Baute et al., Phys. Rev. A 61, 022118 (2000).
[70] V. Delgado and J.G. Muga, Phys. Rev. A 56, 3425 (1997).

[71] I.L. Egusquiza and J.G. Muga, Phys. Rev. A 61, 012104 (1999).

[72] N. Grot, C. Rovelli, and R.S. Tate, Phys. Rev. A 54, 4676 (1996).

[73] J.G. Muga and C.R. Leavens, Phys. Rep. 338, 353 (2000).

[74] J.A. Damborenea, I.L. Egusquiza, G.C. Hegerfeldt, and J.G. Muga, Phys. Rev. A 66, 052104 (2002).

[75] G. Grubl and K. Rheinberger, J. Phys. A 35, 2907 (2002).

[76] S. Kreidl, G. Grubl, and H.G. Embacher, J. Phys. A 36, 8851 (2003).

[77] I.L. Egusquiza, J.G. Muga, B. Navarro, and A. Ruschhaupt, Phys. Lett. A 313, 498 (2003).

[78] R. Brunetti and K. Fredenhagen, quant-ph/0103144, arXiv.

[79] G.R. Allcock, Ann. Phys. 53, 311 (1969).

[80] A.J. Bracken and G.F. Melloy, J. Phys. A 27, 2197 (1994).

[81] J.G. Muga, J.P. Palao, and C.R. Leavens, Phys. Lett. A 253, 21 (1999).

[82] R. Brunetti and K. Fredenhagen, quant-ph/ 0207048, arXiv http: / / xxx.lanl.gov

\title{
LAIKO PROBLEMA KVANTINĖJE MECHANIKOJE IR JOS ANALIZE் NAUDOJANT SILPNUS MATAVIMUS
}

\author{
J. Ruseckas, B. Kaulakys \\ VU Teorinès fizikos ir astronomijos institutas, Vilnius, Lietuva
}

\section{Santrauka}

Pateikta laiko problemos kvantinejje mechanikoje analizès silpnu matavimu teoriniu pagrindu apžvalga. Tuneliavimo trukmės problema yra bendresnès laiko problemos kvantineje mechanikoje atskiras atvejis. Problema spręsta laikui matuoti naudojant silpnu matavimu teoriją. Tokiam matavimo modeliui rasta trukmés išraiška (13), kai fizikinis dydis turi konkrečią vertę arba yra tam tikroje verčiu srityje. Tos išraiškos atskiras atvejis yra tuneliavimo trukmè, t. y. trukmè, kurią tuneliuojanti dalelè praleidžia po potencialiniu barjeru.

Nagrinejjama trukmè, kuriai esant fizikinis dydis turi konkrečią vertę su sąlyga, kad sistema yra tam tikroje galinèje būsenoje. Naudojamu matavimo modeliu galima gauti šios trukmès išraišką (15). Taip apibrèžta trukmé turi daug trukmès klasikineje mechanikoje savybių, tačiau ne visuomet turi prasmę. Galima rasti trukmę, kuriai esant fizikinis dydis turi konkrečią vertę su sąlyga, kad sistema yra konkrečioje galinejje būsenoje, tik kai yra patenkinama (19) salyga. Priešingu atveju matavimo rezultatas priklauso nuo konkretaus matavimo būdo, ir vienareikšmis atsakymas yra negalimas. Kai (19) salyga yra netenkinama, galima apibrežti du trukmę api- būdinančius dydžius (16) ir (17). Šie dydžiai gali būti taikomi ir tuneliavimo trukmès problemai nagrinèti.

Tuneliavimo trukmei nagrinèti naudojome Steinberg'o [34] pasiūlytą būdą, kuris parodo operatorių nekomutatyvumo pasekmes ir asimptotinès trukmès vertinimo galimybę. Mūsu modelyje taip pat pasireiškia Hartmann'o ir Fletcher'io efektas: platiems barjerams efektyvus greitis yra labai didelis, nes barjero srities indèlis $\mathfrak{i}$ trukmę yra labai mažas. Ar šis greitis didesnis už šviesos greitị $c$, ívertinti nepavyksta. Tam reikètų spręsti reliatyvistines lygtis.

Pasiūlytas atvykimo laiko iš vienos pusès pasiskirstymo tankio apibrēžimas. Šis apibrěžimas yra išplèstas i kvantinę mechaniką, naudojant silpnus matavimus. Pasiūlytas būdas yra tinkamas tiek laisvoms dalelèms, tiek ir dalelèms, esančioms išoriniame potenciale. Randama ne tik atvykimo laiko pasiskirstymo tankio išraiška, bet ir būdas jam išmatuoti. Tačiau šis būdas neduoda vienareikšmio atsakymo. Analogiškai kompleksinei tuneliavimo trukmei, yra apibrežtas kompleksinis atvykimo laiko pasiskirstymo tankis. Parodyta, kad taikant pasiūlytą metodą, atvykimo laiko nustatymo tikslumas kvantineje mechanikoje yra ribotas. 